\title{
Terahertz Frequency Metrology for Spectroscopic Applications: a Review
}

\author{
L. Consolino $^{1}$ (D) $\cdot$ S. Bartalini ${ }^{1} \cdot$ P. De Natale $^{1}$
}

Received: 13 March 2017 / Accepted: 2 June 2017 /

Published online: 25 July 2017

(C) The Author(s) 2017. This article is an open access publication

\begin{abstract}
We provide an overview on terahertz (THz) frequency metrology, starting from the nowadays available continuous wave $\mathrm{THz}$ sources, discussing their main features such as tunability, spectral purity, and frequency referencing to the primary frequency standards. A comparison on the achieved results in high-precision molecular spectroscopy is given and discussed, and finally, a special emphasis poses on the future developments of this upcoming field.
\end{abstract}

Keywords Terahertz Frequency Metrology · High Precision Spectroscopy · CW THz sourc

\section{Introduction}

Due to the lack of high-power, tunable sources and fast, sensitive detectors, the terahertz (THz) region of the electromagnetic spectrum has been lagging behind from a technological point of view. The lack of suitable technologies led to the THz band being called the "THz gap." This technological gap has been rapidly diminishing during the last three decades, giving rise to scientific opportunities that could not be considered in the past.

$\mathrm{THz}$ radiation has got plenty of attractive applications, among which $\mathrm{THz}$ high-precision molecular spectroscopy has a central role. In this window, in fact, linestrengths of molecular transitions are generally larger than in the microwave region and comparable with the strongest fundamental ro-vibrational transitions in the mid-IR [1]. Moreover, Hz-linewidth transitions represent key molecular signatures and the $\mathrm{THz}$ range can well represent a novel molecular fingerprint region. For this reason, astronomy and space science have recently moved to $\mathrm{THz}$ technology [2]. As a topical example, one half of the total luminosity of the galaxy and $98 \%$ of the photons emitted since the Big Bang fall into the terahertz gap [3]. Much of this radiation is emitted by cool interstellar dust inside our and other galaxies, and thus, the study of the

L. Consolino

luigi.consolino@ino.it

1 INO, Istituto Nazionale di Ottica, CNR, Largo E. Fermi 6, 50125 Florence, Italy 
discrete lines emitted by light molecular species can give nice insight into star formation and decay, despite the clear need of satellite platforms or high altitudes, due to the strong atmospheric absorption resulting from pressure broadened water and oxygen lines. Furthermore, terahertz thermal emission from gases in the stratosphere and upper troposphere such as water, oxygen, chlorine, and nitrogen compounds is useful for the study of chemical processes related to ozone depletion, pollution monitoring, and global warming [4]. Other spectroscopic applications include plasma fusion diagnostics [5] or identification of different crystalline polymorphic states of a drug. In this spectral range, natural transition linewidths can be as narrow as a few hertz; therefore, it is crucial to probe them with narrow-emission tunable sources, which have been studied throughout the years.

Originally, incoherent thermal sources and Fourier-transform spectrometers were used to probe $\mathrm{THz}$ rotational transitions, requiring cryogenic bolometric detection. In order to achieve high resolution, inconveniently long mirror travel lengths were required and, therefore, obviously the need for a coherent source arose in this spectral range. The aim of this essay is to review the characteristics, the results, and the future perspectives of high-precision $\mathrm{THz}$ spectroscopy based on continuous wave (CW) sources.Free electron type sources like Klystrons, Traveling Wave Tubes (TWT), Backward Wave Oscillators (BWO), and Gyrotrons have been extensively studied since the mid of the past century to approach the high frequency part of the microwave region. Apart from Gyrotrons, which are generally designed to work in pulsed mode and to reach high average power, the above free electron sources suffer from simple physical scaling problems, metallic wall losses, and the need for high magnetic and electric fields, as well as high-electron current densities as the frequency is increased. For this reason, TWTs usually do not reach frequencies higher than $100 \mathrm{GHz}$, while BWOs, which can be tuned over tens of gigahertz by varying the accelerating potential, can cover the frequency range extending from $30 \mathrm{GHz}$ to $1.2 \mathrm{THz}$.

BWOs, actually, have been successfully used to perform precise $\mathrm{THz}$ spectroscopy [6]. For example, Winnewisser et al. [7] used tunable narrow-linewidth $(<20 \mathrm{kHz})$ backward-wave oscillator (BWO) to perform sub-Doppler saturation-dip spectroscopy of rotational transitions of CO to reveal the upper limit for the Doppler-free linewidths of $16 \mathrm{kHz}$ at $230 \mathrm{GHz}, 25 \mathrm{kHz}$ at $461 \mathrm{GHz}$, and $32 \mathrm{kHz}$ at $691 \mathrm{GHz}$.

In this framework, however, we will focus on table-top sources, which can be ascribed to three main scenarios: (i) direct $\mathrm{THz}$ lasing action, (ii) frequency up-conversion of microwaves in solid-state diodes, and (iii) frequency down-conversion of visible/infrared light in non-linear media and photoconductive antennas, and of course hybrid setups that will also be discussed. We will start by reviewing the recent history of the three scenarios, giving particular importance to emission power, tunability, and spectral purity achieved by the different sources. Particular importance will be given to the demonstration and characterization of free-standing $\mathrm{THz}$ frequency comb synthesizer (FCS), and then we will review the results obtained by these setups and finally give out perspectives on possible future developments and applications.

\section{Sources}

\subsection{Direct THz Lasing Action}

The need for a coherent THz source was first satisfied by optically pumped Far-InfraRed (FIR) lasers. This kind of source provided high enough power levels, but was very limited in 
tunability ranges, being based on the emission of a finite set of discrete molecular transitions. Extended tunability has been achieved with the tunable far-infrared spectrometer (TuFIR) hybrid approach discussed in Sect. 2.3.

In an optically pumped FIR laser, the molecular gas is excited from its ground vibrational state to a higher vibrational state by an external laser source. Population inversion is then obtained between two rotational levels within the excited vibrational state. The pump source must be tuned so that its wavelength closely coincides with an absorption line in the molecular gas. Typically, the molecular absorption bands lie in the $10 \mu \mathrm{m}$ region $(0.1 \mathrm{eV})$. All infrared emitting lasers are potential candidates for FIR laser pumping, provided that the FIR laser medium absorbs the pump wavelength. Carbon dioxide $\left(\mathrm{CO}_{2}\right)$ lasers are powerful emitters of coherent radiation at a large number of discrete wavelengths around $10 \mu \mathrm{m}$. Since typical molecular absorption bands lie within the $\mathrm{CO}_{2}$ laser spectrum, FIR laser pumps are almost exclusively $\mathrm{CO}_{2}$ lasers. The energy difference between two rotational levels within one vibrational state is typically $0.01 \mathrm{eV}$, which is the energy of FIR photons. Stimulated Raman emission is also a contributor to the richness of FIR laser lines, especially at high pump power levels. However, even at low pump powers, laser emissions that cannot be assigned to a specific transition in the FIR laser medium are attributed to stimulated Raman. A vast number of polyatomic molecules have been found to lase when optically pumped by infrared lasers. Heavy molecules preferentially produce longer wavelengths. For example, formic acid, $\mathrm{HCOOH}$, lases at longer wavelengths than the lighter methyl alcohol, $\mathrm{CH}_{3} \mathrm{OH}$. The heavier $\mathrm{CF}_{2} \mathrm{Br}$, pumped by a $\mathrm{CO}_{2}$, laser yields the long FIR laser wavelength of $2140 \mu \mathrm{m}(140 \mathrm{GHz})$. Despite the abundance of observed FIR laser lines, only a limited number of them are powerful enough to be used in practical applications. Usually, a FIR laser molecule only produces a few powerful laser lines. Due the complexity of its FIR molecular spectrum, and the consequent richness of FIR laser lines, methyl alcohol has become a favorite FIR laser medium. Normal (non-isotopic) $\mathrm{CH}_{3} \mathrm{OH}$ alone is responsible for more than $300 \mathrm{FIR}$ laser lines, ranging from 30 to $1200 \mu \mathrm{m}$. In methyl alcohol, the overlap between the strongly absorbing CO-stretch band and the $\mathrm{CO}_{2}$ laser spectrum is excellent; the $\mathrm{CH}_{3} \mathrm{OH}$ molecule shows fairly large permanent electric dipole moments both parallel and orthogonal to the axis of symmetry. Exchanging $\mathrm{H}$ by $\mathrm{D}$ in the $\mathrm{CH}_{3} \mathrm{OH}$, thereby obtaining the deuterated species $\mathrm{CH}_{3} \mathrm{OD}$, $\mathrm{CH}_{2} \mathrm{DOH}, \mathrm{CHD}_{2} \mathrm{OH}, \mathrm{CD}_{2} \mathrm{OH}$, and $\mathrm{CD}_{3} \mathrm{OD}$, does not shift the CO-stretch band appreciably; the absorption still falls within the $\mathrm{CO}_{2}$ laser spectrum. On the other hand, different FIR laser lines do appear since the rotational and vibrational levels are affected. It is also possible to exchange the ordinary ${ }^{12} \mathrm{C}$ carbon atom by the ${ }^{13} \mathrm{C}$ isotope.

In general, using an FIR laser involves bulky instrumentation, sophisticated operation, and is intrinsically limited by the active media transition frequencies. Consequently, availability of a new generation of compact, reliable $\mathrm{THz}$ sources is the key for the development of the largely underdeveloped $\mathrm{THz}$ range. In this framework and THz-emitting Quantum Cascade Lasers (QCLs) are proving to be good candidates to fill this gap. For a dimension comparison between QCLs and molecular lasers, see Fig. 1. The first report on QCLs operating at THz frequencies is relatively recent [8] and, despite the cryogenic operation temperatures $(<199 \mathrm{~K})$ [9], THz QCLs have attracted considerable attention thanks to the high output power (>100 mW), spectral purity, stability, compactness, and reliability, and have now a realistic chance of making a deep impact on technological applications. Among the features that a $\mathrm{THz}$ QCL has to show to become a useful radiation source for many applications is a reliable, repeatable, and tunable single-frequency emission. Many quantum-design related approaches, technological solutions, and/or optical configurations have been indeed recently tested to tune the emission frequency of $\mathrm{THz}$ QC sources over quite a large bandwidth [10]. 
Fig. 1 Comparison between the bulky instrumentation of a molecular FIR laser, whose cavity length is in the order of $2 \mathrm{~m}$, and a THz Quantum Cascade Laser, whose dimensions are in the millimeter range

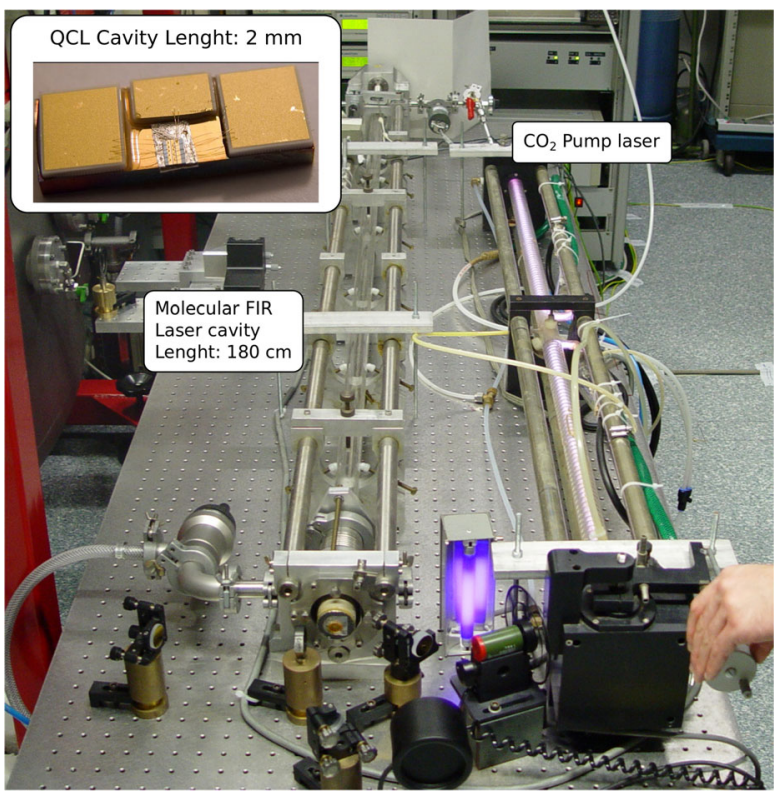

Design and fabrication of such long wavelength QC lasers have been challenging: the emission, at energies lower than the optical phonon one, makes e-e scattering a non-negligible energy relaxation channel; the huge free-carrier absorption becomes the dominant factor of the waveguide losses; anomalies in semiconductor dielectric constant further complicate laser action and must be taken into account for designing optical waveguides that overcome the high losses and the practical limitations induced by prohibitive layer thickness; the non-radiative lifetimes ( $\sim 0.5 \mathrm{ps})$ of intersubband transitions become very short, and carriers tend to enhance the leakage channels. Also, it is important to stress that, at terahertz frequencies, the spontaneous emission lifetime is typically much longer (microseconds) compared to the nonradiative lifetimes (picoseconds), so that radiative relaxation does not play any role in transport below threshold.

At this time, three active region types have successfully been used in terahertz QCLs: chirped superlattice, bound-to-continuum, and resonant phonon. In addition, a combined scheme of bound-to-continuum and resonant phonon, namely interlaced photon-phonon cascade, has been also developed. The first report on a THz QCL [11] exploited a careful design of the active region, based on a chirped superlattice and an asymmetric low-loss waveguide, and emission at $4.4 \mathrm{THz}$ was achieved.

The gain spectrum of THz QCLs can be engineered by quantum design to be broadband over a range even larger than $1 \mathrm{THz}$, or, alternatively, to be electrically tunable over a $240-\mathrm{GHz}$ bandwidth, exploiting the Stark shift of the lasing transition.

For reliable and repeatable single-mode tuning, QCL emitters based on external cavities can be realized using either a movable mirror to control the cavity length or a rotating grating for wavelength-dependent feedback. First-order and second-order DFB THz QCLs also provide stable single-mode emission, via temperature/current tuning, though usually on a more limited range, of only a few gigahertz. However, if coupled to an external microcavity, wider tuning ranges $(\approx 20 \mathrm{GHz})$ can be achieved by mechanically changing the size of the external 
microcavity. As an alternative, the lateral size of the lasing mode can be manipulated in narrow $(\sim \lambda / 3)$ ridge, double-metal $\mathrm{THz}$ DFBs via electromechanical components, to tune the laser emission widely and continuously on a bandwidth of over $330 \mathrm{GHz}$ [12].

Another surprising characteristic of THz QCLs is their spectral purity. In fact, frequencyand phase-stabilized, high-power, and reliable solid-state terahertz sources can find application in a number of fields, spanning from far-IR astronomy [13] and high-precision molecular gas spectroscopy [14] to high-resolution coherent imaging and telecommunications $[15,16]$. In this context, knowledge of the intrinsic linewidth, ultimately related to the uncertainty principle of quantum mechanics, plays a key role, determining the maximum achievable spectral resolution and coherence length in a free-running laser. Recently, the spectral purity of a $\mathrm{THz}$ QCL has been investigated via the measurement of its frequency-noise power spectral density (FNPSD), providing an experimental evaluation and a theoretical assessment of its intrinsic LW. Intensity measurements were performed to retrieve information in the frequency domain by converting the laser frequency fluctuations into detectable intensity (amplitude) variations [17]. These measurements led to a measured FWHM $\delta v=90 \pm 30 \mathrm{~Hz}$ [18]. This result has been later independently confirmed [19].

Experiments on frequency and phase stabilization of THz QCLs have involved right from the beginning some secondary frequency standards. In 2005, phase-lock of a 3-THz QCL to the $3.1059368 \mathrm{THz}$ line of a methanol gas laser was demonstrated [20] and, in 2009, frequency stabilization of a single-mode THz QCL against the $2.409293 \mathrm{THz}$ line of a $\mathrm{CH}_{2} \mathrm{DOH}$ gas laser was obtained [21]. In both cases, a narrowing of the beat signal was observed ( 65 and $3 \mathrm{kHz}$, respectively). The first THz QCLs locked to microwave-driven harmonically generated $\mathrm{THz}$ sources were demonstrated at $1.5 \mathrm{THz}$ [22], $2.7 \mathrm{THz}$ [23] (phase locks), and 2.3 THz [24] (frequency lock). Despite the high complexity, the high electrical power consumption, and the very low efficiency with a few $\mathrm{pW}$ radiation powers, such kinds of sources provide a very narrow and absolute-frequency reference, suitable for mixing with the THz QCL in a sensitive detector such as a hot-electron bolometer. Frequency-locking of a THz QCL to a molecular reference was demonstrated in a first-derivative, direct absorption spectroscopy configuration, without any significant narrowing of the QCL emission [25-27]. Phase-locking to an optical FCS (OFCS) was achieved only recently [28] by locking a 2.5-THz QCL to the $n$th harmonic of the repetition rate of a mode-locked erbium-doped fiber laser. By implementing a technique first reported by Löffler et al. [29], the comb generated by the mode-locked laser was mixed in a non-linear crystal with a CW THz QCL, thus generating THz sidebands around the near-IR carrier. The beating between the original comb and its shifted replicas provides the signal for closing the phase-lock loop. A similar scheme, but using a photoconductive antenna instead of electrooptic detection, was presented 1 year later [30].

Despite the clear advantage of room temperature detection of the beat-note, the latter approaches are based on low-efficiency up-conversion processes, and they inherently require a CW THz power in the milliwatt range. This can represent a limitation in many cases, when enough output power is not available or when it is needed for other simultaneous applications (i.e., spectroscopy). One possible solution has been provided by moving to a $\mathrm{THz}$ detection, where the beat-note can be acquired by a square-law $\mathrm{THz}$ detector with much higher efficiency, therefore involving only a small fraction of the overall emitted QCL power. Of course, this detection involves a free-standing and air-propagating $\mathrm{THz}$ FCS.

Coherent THz pulses are largely employed in time-domain spectroscopy, and even though this review focuses on $\mathrm{CW}$ sources, a focus on $\mathrm{THz}$ comb is needed for their importance in narrowing and referencing THz QCLs. In fact, the intrinsic comb nature of pulsed THz sources 
used in time-domain spectroscopy has been recently demonstrated [31,32], and direct use of such sources as frequency "ruler" for a THz QCL has ever been reported in 2012, by phaselocking a single-frequency CW QCL emitting at $2.5 \mathrm{THz}$ onto a single tooth of a THz FCS [33]. This allowed for not only the direct knowledge of the QCL absolute frequency, but also a narrowing of its emission linewidth down to around $130 \mathrm{~Hz}$ could be observed.

In [33], the $\mathrm{THz}$ comb generation is realized by optical rectification, in Cherenkov configuration [34], of a femtosecond mode-locked Ti/Sa laser in a single-mode waveguide. The waveguide is fabricated on a $\mathrm{MgO}$-doped $\mathrm{LiNbO}_{3}$ crystal plate [35]. The generated radiation is a train of coherent $\mathrm{THz}$ pulses, carrying a very large spectral content (from few $\mathrm{GHz}$ up to more than $6 \mathrm{THz}$ ). Since the pulses are identical, the comb-like spectrum of the infinite train has a perfectly zero offset, and a spacing corresponding to the repetition rate of the pump laser, which was stabilized against a Rb-GPS (Global Positioning System) disciplined $10 \mathrm{MHz}$ quartz oscillator. Stability in the megahertz range was obtained for the repetition rate, and this ensures stability of each tooth of the THz comb at the $100 \mathrm{~Hz}$ level, depending on the order of the tooth.

\subsection{Frequency Up-conversion of Microwaves in Solid-State Diodes}

Coming from the electronics frequency range, planar Schottky diodes based on frequency multiplier chains pumped at W-band with $100-150 \mathrm{~mW}$ have reached $1.9 \mathrm{THz}$ in 2004 [36-38] with sufficient power to be used as a LO for HEB mixers [39, 40]. These chains produce from several to tens of microwatts at room temperature but, as reported in an earlier work [41], they greatly improve upon cooling at $120-150 \mathrm{~K}$ and in 2005 , a $2 \times 3 \times 3$ multiplier chain reached a record $100 \mu \mathrm{W}$ at $1.665 \mathrm{THz}$ [42]. Moreover, they are electronically and continuously tunable over $10-15 \%$ of their bandwidth, and they are intrinsically easy to be referenced to primary frequency standards, in order to precisely know their emission frequency.

In the 1990s, frequency multipliers based on whisker-contacted Schottky diodes played an important role in the development of heterodyne receivers for radio astronomy as they appeared to be the only available solution for the LOs of space-borne submillimeter-wave heterodyne instruments. However, in 1992, this already mature technology was still unable to pass the $1 \mathrm{THz}$ milestone [43]. Progress toward the terahertz region was reported by Crowe and Rüdiger Zimmerman [44] just before Zimmerman reached $1.135 \mathrm{THz}$ in 1998 with an allsolid-state source that produced $40 \mu \mathrm{W}$ of output power [45].

At submillimeter wavelengths and until the year 2000, whisker-contacted diodes outperformed Schottky planar diodes introduced in the mid-1980s by Cronin and Law [46] at the University of Bath, UK, and shortly later by Bishop and Mattauch [47] at the University of Virginia (UVa), USA. However, at millimeter-wavelength Schottky planar discrete diodes started to give better performance due to the use of multiple anodes in balanced configurations. Erickson's balanced doublers, proposed and demonstrated in [48-50], have become a standard topology for frequency multiplication due to their good performance. Gallium arsenide (GaAs)-based heterostructure barrier varactors (HBVs) were introduced by Kollberg and Rydberg at the University of Chalmers [51], Sweden, as alternate diodes, and this technology took a significant turn in the late 1990s when Lippens and Mélique at IEMN, France, introduced indium phosphide ( $\mathrm{InP}$ )-based multiple barrier devices [52]. The results obtained with a $250-\mathrm{GHz}$ waveguide tripler (11\% efficiency and $9.5 \mathrm{~mW}$ of output power) demonstrated that HBV technology was a serious challenger to the classic and simpler Schottky technology. 
However, despite further efforts by IEMN, Chalmers, and UVa, HBV multipliers did not reach the level of performance of Schottky multipliers. Another technique to build devices that exhibit internal symmetries was explored by Krach [53]. It gave a conversion efficiency of $22 \%$ for a $230-\mathrm{GHz}$ planar diode tripler.

In the mid-1990s, the release of powerful commercial three-dimensional (3D) field-solvers (Ansoft HFSS) and non-linear circuit simulators (HP-now-Agilent MDS-now-ADS) transformed the way frequency multipliers were designed and built. These codes greatly increased the accuracy and the speed of the calculations necessary to optimize frequency multipliers. Erickson and Tiovunen pioneered the design of a four-anode balanced doubler at $170 \mathrm{GHz}$, entirely with HFSS and MDS [54]. Within the years 1995-2000, it became clear that discrete planar diodes were limited in frequency due to their size and the difficulty to connect them to the circuit with sufficient precision. Their integration on a circuit featuring several matching elements and providing precise connections to the waveguide block was necessary. The precision of the machining of the waveguide blocks plays a fundamental role in the working of THz frequency multipliers. For instance, JPL 1.9 THz tripler chips are inserted in a channel which width and depth are respectively 38 and $12 \mu \mathrm{m}$. The required precision for the alignment of the chip in the channel or the alignment of the two halves of the block is 2 to $3 \mu \mathrm{m}$.

These sources have recently been demonstrated and characterized for $\mathrm{THz}$ frequencies up to $2.75 \mathrm{THz}$ [55-58], with output powers in the 1-14 $\mu \mathrm{W}$ range, as can be seen from Fig. 2. This source is based on a 91.8- to 101.8-GHz synthesizer followed by a power amplifier and three cascaded frequency triplers. It demonstrates, for the first time, that purely electronic solid-state sources can generate a useful amount of power in a region of the electromagnetic spectrum where lasers (solid state or gas) were previously the only available coherent sources.

\subsection{Frequency Down-conversion of Visible/Infrared Light in Non-linear Media and Photoconductive Antennas}

Historically, frequency conversion by non-linear generation has been a key tool for synthesizing radiation, often continuously tunable over wide intervals, in regions where lasers did not directly emit. Non-linear difference frequency generation has been conveniently used for many years to generate $\mathrm{cw}$ radiation throughout the infrared region, till the millimetric range, where microwave sources had been available as coherent sources before the advent of the laser.
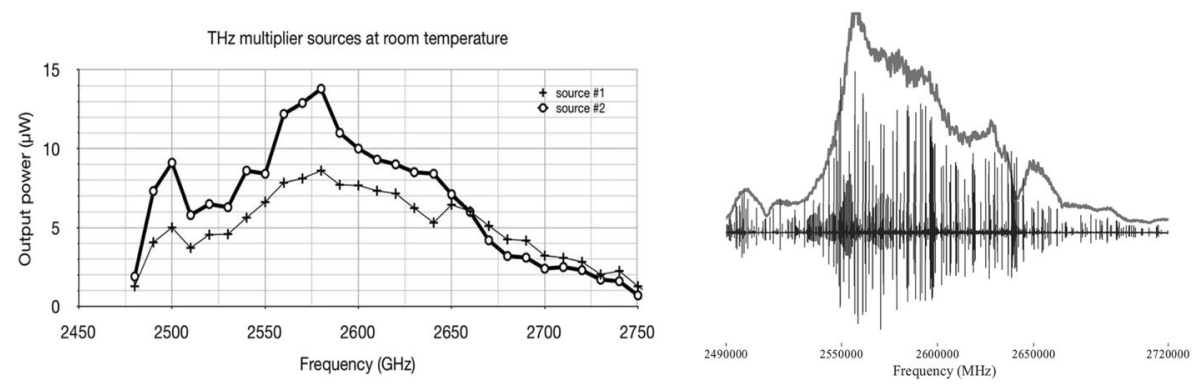

Fig. 2 Frequency up-conversion setups have been successfully demonstrated to frequencies up to $2.77 \mathrm{THz}$, and output powers in the $10-\mu \mathrm{W}$ range. Calibrated output power spectrum measured for $2 \mathrm{THz}$ multiplier sources in the range $2.475-2.750 \mathrm{THz}$, at room temperature (left). Amplitude modulated power spectrum of the multiplier source (gray trace), and second derivative tone burst modulated spectrum of methanol vapors (black trace) (right). Reprinted with permission from [56], copyright 2011 
In the 1980s, two metrological-grade techniques, based on difference frequency generation by non-linear conversion, were developed. The first of these is generation of microwave sidebands on the strongest FIR laser lines in Schottky diodes [59-62]. It produces tunable FIR radiation up to about $3 \mathrm{THz}\left(100 / \mathrm{cm}^{-1}\right)$ with $5 \mathrm{kHz}$ linewidths and roughly $500 \mathrm{kHz}$ accuracy. Thanks to the pioneering work of K.M. Evenson and co-workers, a significant improvement in accuracy and tunability range were obtained with the tunable far-infrared spectrometer (TuFIR) [63]. It is based on a synthesis of FIR radiation starting from infrared and microwave radiation mixed onto a non-linear device, namely the metal-insulator-metal (MIM) diode. The MIM diode consists of an electrochemically sharpened tungsten whisker (25-pm diameter and 3 to $7 \mathrm{~mm}$ long) contacting a metal base. The metal base has a naturally occurring thin-oxide insulating layer. Both nickel and cobalt have been used as base materials, but cobalt is generally more consistent in the production of third-order FIR radiation. In a later version [64], the TuFIR spectrometer produces radiation through non-linear mixing of three radiations (two from $\mathrm{CO}_{2}$ lasers and one from a microwave source; see Fig. 3) in a third-order MIM diode $[65,66]$. The MIM diode generates microwave sidebands on the $\mathrm{CO}_{2}$ difference frequency. The standard frequency coverage of such a source is from 0.3 to $6 \mathrm{THz}$; the lower limit is set by the bolometer and the upper limit by the largest difference frequency between the two $\mathrm{CO}_{2}$ lasers. The MIM diode was for many years a key device for frequency metrology, and it is interesting to compare its properties with present-day photoconductive (PC) switches for photomixing observation.

CW THz generation by photomixing is based on the THz-periodic generation of electrons and holes in semiconductors by absorption of two interfering laser beams of frequencies $v_{1}$ and $v_{2}$, for which $v_{\mathrm{THz}}=v_{2}-v_{1}$. The interference results in intensity modulation of the laser beams with the frequency $v_{\mathrm{THz}}$ and, hence, the THz-periodic carrier generation. The emission of $\mathrm{THz}$ radiation originates either directly from the (phase-coherent) acceleration of the

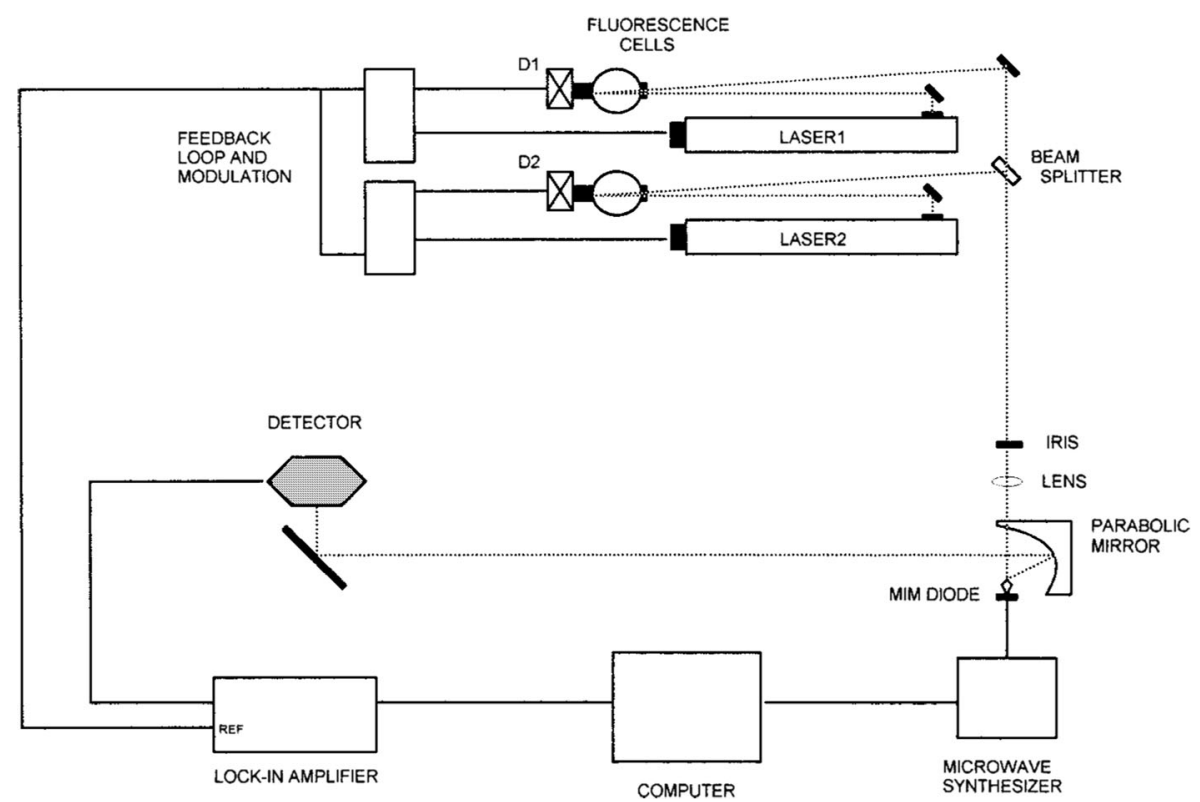

Fig. 3 Schematic view of a typical TuFIR spectrometer. Reprinted with permission from [66], copyright 1998 
individual photogenerated carriers or from the collective current of these carriers, generated within a very small area and being fed into an antenna.

Low temperature (LT) GaAs is the prevailing PC antenna material for this technique because of its high mobility and short lifetime. A photomixer is a compact, solid-state device. The tuning range can be exceptionally broad provided that a high-quality, tunable, dual-frequency laser system is available. The primary disadvantage of this method is that the output power is relatively low compared with other techniques of $\mathrm{CW} \mathrm{THz}$ generation. Its optical-to- $\mathrm{THz}$ conversion efficiency is $10^{-6}-10^{-5}$, and the typical output power is in the microwatt range. Because photomixing requires continuous optical excitation, the maximum $\mathrm{THz}$ output power is limited by the low thermal conductivity of LT-GaAs $(\sim 15 \mathrm{~W} / \mathrm{mK})$. The damage threshold of a 1- $\mu \mathrm{m}$ LT-GaAs layer with biased electrodes is less than $10^{5} \mathrm{~W} / \mathrm{cm}^{2}$ of optical excitation [67].

A typical photomixer includes an antenna structure of metal on a LT-GaAs layer grown on a SI-GaAs substrate. A silicon hyper-hemispherical lens is attached to the back side of the substrate. A commonly used antenna structure for photomixing is the logarithmic uniplanar spiral antenna (log-spiral antenna) with interdigitated electrode fingers [68]. It has the advantage of a broad tuning range: its radiation pattern, impedance, and polarization remain virtually unchanged below $1 \mathrm{THz}$. The carrier lifetime of LT-GaAs and the RC time constant of a typical log-spiral photomixer are a few hundred femtoseconds; thus, frequency roll-off begins around $1 \mathrm{THz}$. The generated radiation power is proportional to $\omega^{-4}$ at higher frequencies. At the expense of a broad tuning range, the log-spiral antenna has low output power due to the relatively low radiation resistance. The radiation power can be enhanced by using resonant antenna structures [69]. The radiation resistance of a dipole antenna on a GaAs substrate peaks around $L / \lambda_{\mathrm{R}}=0.3$ [70], which corresponds to the resonant frequency $\nu_{\mathrm{R}} \approx 1.8 \mathrm{THz}$ for the $50-\mu \mathrm{m}$ dipole. The measured output power is maximized near $1.2 \mathrm{THz}$ and extends throughout the broad spectral range from 0.5 to $2 \mathrm{THz}$.

Higher output powers are achievable using more sophisticated antenna designs, such as dual dipole antennas. The advantages over simpler antenna designs include a more symmetric radiation pattern and a higher radiation resistance [71]. In this design, the electrode capacitance is canceled out by the inductive tuning when the length of the transmission line is adjusted to the resonant frequency. Thus, the radiation resistance is determined mainly by the carrier lifetime. The output spectra of the dual-dipole antennas demonstrate significantly higher powers near their resonant frequencies when compared to the output from the log-spiral antenna [71]. The maximum output powers of the dual-dipole antennas are 3, 2, 0.8, and $0.3 \mu \mathrm{W}$ at $0.9,1.0,1.6$, and $2.7 \mathrm{THz}$, respectively. The peak power declines with increasing frequency as $\omega^{-2}$, while the log-spiral output is proportional to $\omega^{-4}$.

In order to avoid the limitation due to the low threshold for thermal damage of LT-GaAs, as well as to increase photocurrent, the optical excitation area can be made larger and can be illuminated with an extended beam from a high-power laser. If the dimensions of the illumination area are comparable to or larger than the wavelength of radiation, the optical excitation should be phase-matched with the THz radiation [72]. Another approach to enhance photocurrent is to replace LT-GaAs substrates with semiconductor heterostructures such as $\mathrm{p}$-i$\mathrm{n}$ photodiodes. A unitraveling-carrier photodiode (UTC-PD) contains a collection layer of InP which takes advantage of the exceptionally high-electron mobility in the material and of the optical excitation at the optical communication wavelength, $1.55 \mu \mathrm{m}[73]$.

In order to overcome the limitations set by the finite carrier lifetime of semiconductor materials that induce a pronounced roll-off at higher frequencies, limiting the available $\mathrm{THz}$ power beyond $1 \mathrm{THz}$ to a few microwatts, the approach of difference frequency generation 
(DFG) in non-linear crystals can be followed. DFG is a second-order non-linear optical process; thus, it requires a non-centrosymmetric crystal. Like photomixing, the optical source for DFG are two narrowband laser beams with slightly different frequencies $v_{\mathrm{THz}}=v_{2}-v_{1}$. In these DGF operations, phase matching is crucial for efficient $\mathrm{THz}$ generation, and therefore, various phase matching methodologies have been developed, such as birefringence and quasiphase matching, using periodically poled crystals or Cherenkov emission schemes.

Regarding the materials used, various crystals have been explored for THz DFG, starting with simple bulk configurations in inorganic materials, such as lithium niobate $\left(\mathrm{LiNbO}_{3}\right)$ [74, 75], lithium tantalate $\left(\mathrm{LiTaO}_{3}\right)$ [75, 76], zinc telluride ( $\left.\mathrm{ZnTe}\right)$ [77, 78], indium phosphide (InP) [79], gallium arsenide (GaAs) [80], gallium selenide (GaSe) [81, 82], cadmium telluride ( $\mathrm{CdTe}$ ) [83], cadmium zinc telluride (CdZnTe) [84], and gallium phosphide (GaP) [85]. The main limitation of these sources relies on the low power achieved, due to the low efficiency of the second-order phenomenon implied in the generation. In order to improve the overall generation efficiency, different experimental setups have been tested. One favorable option is to enclose the generation crystal into an optical cavity for the visible/near IR radiation. These setups can achieve output powers of a few milliwatts at $1.9 \mathrm{THz}$ with intracavity power as high as $500 \mathrm{~W}$ [86]. The generated power scales with the product of the intensities of the two pump beams; as a consequence, the emitted $\mathrm{THz}$ power can be also enhanced by confining the $\mathrm{THz}$ radiation in a small space. To this purpose, ridged [87] and implanted (Garcia Lopez et al., submitted for publication) waveguides have been realized, achieving efficiencies of $10^{-9}$ in the THz generation.

Recently, also organic crystals have been used even if their breakdown power is usually much lower. These organic crystals are ideal for ultrawide band operation, and include 4dimethylamino- $N$-methyl-4-stilbavolium tosylate (DAST) [88-92] or $\mathrm{N}$-benzyl-2-methyl-4nitroaniline (BNA) [93, 94].

Another important kind of source that relies on $\chi^{2}$ processes is the optical parametric oscillator (OPO), which has recently been successfully used for $\mathrm{THz}$ generation. For example, CW THz output tunable between 1.3 and $1.7 \mathrm{THz}$ was produced in a cascaded parametric process, where the resonantly enhanced near infrared "signal" wave of a primary 1030-nm pumped periodically poled lithium niobate (PPLN) OPO served as pump for the secondary OPO (using the same PPLN crystal and the same optical cavity) with the backward $\mathrm{THz}$ wave as the "idler" [95]. In another setup, based on a pump enhancement cavity with a finesse of 500 at the $1030 \mathrm{~nm}$ pump wavelength, the pump radiation was directly converted to $\mathrm{THz}$, via an OPO process using PPLN, with tunability from 1.2 to $2.9 \mathrm{THz}$ [96]. In both cases, the output power was limited to few microwatts due to strong $\mathrm{THz}$ absorption in lithium niobate.

Intracavity $\mathrm{THz}$ generation using a doubly resonant optical parametric oscillator (DR-OPO) for CW operation was first proposed and implemented in [97], where a quasi-phase-matched (QPM) OP-GaAs crystal was placed inside the cavity of an OPO, designed for type II parametric interaction (signal and idler orthogonally polarized) to allow narrow linewidths when operating near degeneracy. In the DR-OPO, both the signal and the idler waves resonate and the output $\mathrm{THz}$ power scales as cavity finesse squared. The power levels achieved were in the order of $>10 \mu \mathrm{W}$, tunable over the $1-4.5 \mathrm{THz}$ range.

Regarding the spectral purity of DFG sources, it is quite straightforward that the linewidth of the emitted $\mathrm{THz}$ radiation depends on the linewidth and on the phase relation between the pumping sources. For this reason, the advent of optical FCS in the visible/near IR region allowed to transfer the spectral properties of the finest frequency standards directly to the generated DFG radiation. 


\section{High-Resolution Spectroscopy}

In the THz spectral region, most rotational transitions of light molecules fall, which are of high interest for applicative research (mainly astrophysics and atmospheric physics) as well as for fundamental studies. Moreover, since absorption coefficients normally scale as either the square or the cube of frequency for $\mathrm{h} v \ll \mathrm{kT}$, $\mathrm{THz}$ transitions are much more sensitive tools (compared to millimetric transitions) to detect neutral/ionized atoms/molecules. In spite of the very large scientific interest, high-resolution spectroscopic studies in the submillimetric range started much later than in the neighboring microwave and infrared spectral regions, due to the lack of both adequate coherent sources and detectors.

The introduction of optically pumped, fixed frequency Far-InfraRed (FIR) lasers and of liquid-He cooled bolometric detectors (with typical Noise Equivalent Powers-NEP in the range $10^{-12}-10^{-14} \mathrm{~W} / \mathrm{Hz}^{1 / 2}$ ) was the first step toward the development of high-resolution spectroscopy in this region. Laser magnetic resonance (LMR) spectrometry achieves a wider tunability; however, such technique only works on paramagnetic species, tuning the transition of interest into resonance with an FIR laser line by a magnetic field. In this range, LMR proved to be one of the most sensitive spectroscopic techniques, because it is intracavity in nature, with a minimum detectable absorption in the order of $10^{-10}$. Nevertheless, the accuracy of frequency measurements is limited to only several $\mathrm{MHz}$, due to the uncertainty in the extrapolation at zero magnetic field and the lack of reproducibility in the center of the FIR laser line.

The first measurements in a broad range of the $\mathrm{THz}$ region were performed using a Tunable Far Infrared (TuFIR) spectrometer. In this setup, both $\mathrm{CO}_{2}$ lasers are frequency stabilized onto a 4.3- $\mu \mathrm{m}$ wavelength saturated fluorescence signal from low-pressure $\mathrm{CO}_{2}$ cells [98]. These frequencies have been measured with an accuracy better than $5 \mathrm{kHz}[99,100]$. A stability of about $25 \mathrm{kHz}$ is obtained for each locked $\mathrm{CO}_{2}$ laser, and the overall (statistics + systematic) frequency uncertainty of the generated FIR radiation was estimated to be $35 \mathrm{kHz}$ at the best. The spectrometer sensitivity is limited by the FIR power and the sensitivity of the detector. FIR powers up to a few hundreds of nanowatts are generated with $150 \mathrm{~mW}$ from each laser and 6$10 \mathrm{dBm}$ of microwave power applied to the MIM diode. For the best contacts, minimum detectable absorptions are around $10^{-4}$ in a 1 -s integration time, corresponding to a minimum detection coefficient of $10^{-6}$ for a 1 -m long cell. With such a spectrometer, mainly operated at NIST, Boulder, CO, USA, at LENS, Florence, Italy, and later on in Japan [101], a large number of rotational and ro-vibrational transitions of light molecules and free radicals were measured with high accuracy [64, 102-113]. In addition, atomic fine structure transitions could be measured [114-116], as well as transition dipole moments of molecules of atmospheric interest, with an accuracy up to $10^{-4}[117,118]$.

In spite of the scarce reproducibility of MIM diodes fabrication, pioneering studies on the breakdown of the Born-Oppenheimer approximation could be systematically performed as well as a careful analysis of the TuFIR sensitivity [119]. Sophisticated setups could be used notwithstanding the low emitted powers, such as Faraday spectroscopy, based on the measurement of tiny birefringence induced in a sample gas by a longitudinal magnetic field (from which the name: Faraday effect) $[120,121]$. Later studies on the limiting bandwidth of the MIM diode proved that frequencies beyond $9 \mathrm{THz}$ could be generated by using laser lines belonging to rare isotopes of $\mathrm{CO}_{2}$ [122].

Down-conversion generation setups have in principle great potential for broadband frequency metrology applications but, to our knowledge, this field has not been fully developed, 
yet. The groups of Deninger and Sasaki have proposed an InGaAs photomixers and a GaP-based setup, respectively, achieving a resolution of 1 or $15 \mathrm{MHz}[123,124]$. Only very recently, in Florence, a waveguided lithium niobate-based setup has been mounted to get a broadly tunable $\mathrm{THz}$ source, covering the 1 to $8 \mathrm{THz}$ frequency range, which exploits frequency mixing from two tunable CW fiber-amplified telecom lasers in the waveguided crystal. The DFG emission process, confined in a tight $3 \times 5 \mu \mathrm{m}$ section, allows to reach generation efficiencies in the order of $10^{-9} \mathrm{~W}^{-1}$ and power levels in the microwatt range. The source, which provides a $25-\mathrm{GHz}$ modehop-free operation along the whole $7 \mathrm{THz}$ generation span, has also been tested for precision spectroscopy of low-pressure methanol vapors, already proving a relative precision in the order of $4 \times 10^{-9}$ on the line center determination (De Regis et al., submitted for publication).

$\mathrm{THz}$ QCLs are, in principle, suitable candidates for high-precision spectroscopy, and they have already confirmed some expectations. Moreover, setups based on amplitude and frequency modulation have been developed [125]. In particular, in ref. [125], the absolute frequency of the QCL laser was measured with respect to a FIR laser emission, giving an overall accuracy of $8 \times 10^{-7}$ in the line center determination.

As mentioned in the previous section, a great step forward has been achieved with the advent of free standing THz FCSs. The THz comb has been successfully used as local oscillator to narrow and measure the frequency of a THz QCL down to the $10^{-11}$ level [33], only requiring a small fraction of the radiation emitted by the laser. With this setup, highresolution spectroscopy has been performed on single methanol transitions, achieving an accuracy of $4 \times 10^{-9}$ [126]. Figure 4 shows the spectrum acquired for the considered methanol transition, and the measurements performed at different vapor pressures.

At present, the use of a cryogenic detector, such as the hot-electron-bolometer, is probably the main experimental complication of this approach. The combination of a FCS able to cover a broad spectrum (1-6 THz), with QCL sources that can cover, point-by-point, most of the same range but with unprecedented power-levels, is a very promising perspective for a metrological-grade investigation of the THz region. Among the several possible applications, it is worth citing the development of comb-assisted $\mathrm{THz}$ sub-Doppler spectrometers and of absolutely referenced local oscillators for heterodyne THz spectrometers.

Regarding the latter topic, in particular, not only the narrow linewidth of the local oscillator but also, and mostly, the stability over long time periods of its absolute frequency can represent a real breakthrough. A number of demanding new applications will be potentially addressed,

(a)

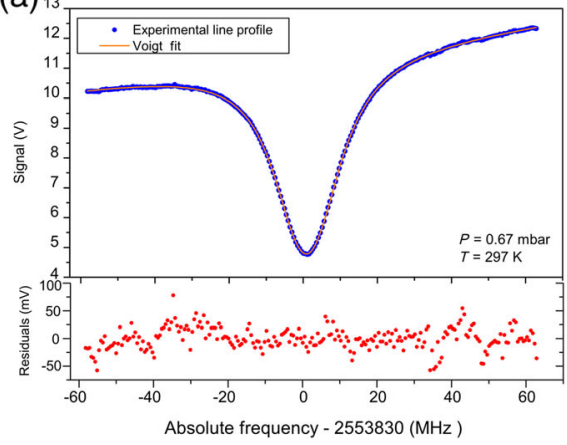

(b)

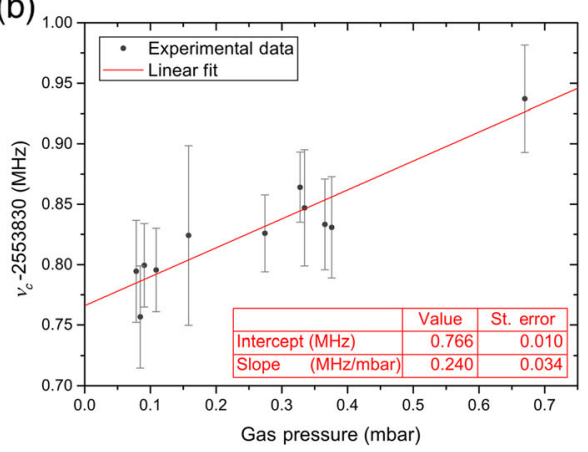

Fig. 4 a Acquisition of a methanol transition around 2.55 , Voigt fit, and residuals. b The same acquisition performed at different vapor pressures. The linear fit gives the transition center with an accuracy of $10 \mathrm{kHz}$. Reprinted with permission from [126], copyright 2014 
e.g., spectroscopic interrogation of cold molecules (see, e.g., [127]), as well as precise measurement of the long-term variation of fundamental physical constants from astronomical observation [128, 129].

Moreover, the development of new high-bandwidth, low-NEP, terahertz detectors, such as nanowire [130, 131] and graphene [132] field-effect transistors, can make possible, in the future, room temperature detection of beat-note signals.

As a matter of fact, the main limitation to the accuracy achieved in [126] is the Dopplerlimited spectroscopy setup used in the experiment. A way to improve the accuracy of the retrieved frequencies has to exploit sub-Doppler resolution, which can be obtained by means of the Lamb-dip technique [133, 134] in case of rotational spectroscopy. Despite the fast growth undergone by spectroscopy and non-linear optics in the past decade, the achievement of sub-Doppler resolution is only at its beginning.

This limitation has been recently overcome by multiplied frequency chain setups, which have achieved an unprecedented accuracy of $1 \times 10^{-9}$ in the line center determination at around $1 \mathrm{THz}$ [135]. Figure 5 shows the comparison between the Doppler limited and the subDoppler acquisition data. A very similar result has been also obtained at much higher frequencies, with an accuracy of $3 \mathrm{kHz}$ on a $2.64-\mathrm{THz}$ line center determination [56]. The bandwidth, agility, and operability of this $\mathrm{THz}$ source have enabled wideband, high-resolution spectroscopic measurements of water, methanol, and carbon monoxide with very high signalto-noise ratio.

\section{Future Perspectives}

Frequency metrology measurements performed on atoms in the visible region achieve a stability of few parts in $10^{-18}$ [136], while measurements performed in the mid-IR on cold molecules are still below $10^{-15}$ [137]. An even wider gap has to be filled for the far IR spectral region. This gap is essentially technological in nature, and continuous efforts are being made by the scientific community to lower the present limit of $10^{-9}[56,126,135]$. Moreover, most of the molecular models, trying to predict molecular transition energies in the THz range, are in the range of $10^{-9}$ relative uncertainty. These models can take into account effects such as

Fig. 5 Comparison between the Doppler broadened line profile and the sub-Doppler acquisition of a $\mathrm{H} 2 \mathrm{~S}$ transition at $1.07 \mathrm{THz}$. Reprinted with permission from [135], copyright 2013, American Chemical Society

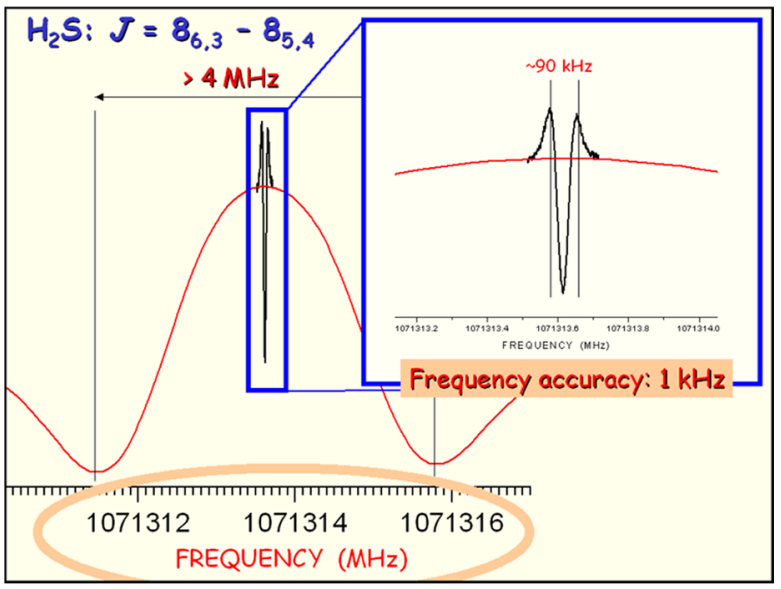


Zeeman shift, AC-Stark shift or blackbody shift, whose contributions are expected to be in the $10^{-10}$ level, and have never been measured before. Systematic observations on a set of relevant molecular transitions would definitely improve the actual knowledge on molecular models and databases. For this reason, new strategies and tools are being developed, some of which will be discussed hereby.

\subsection{Resonant Cavities}

Optical resonators are well-established tools commonly used in spectroscopy [138, 139]. They have widespread applications over the whole electromagnetic spectrum, from microwaves [140] to UV [141], while record-level optical finesse were achieved in the visible/near-IR [142], especially thanks to the advent of whispering gallery mode resonators [143]. In this context, the THz portion of the electromagnetic spectrum is still lacking such tools. Design and fabrication of cavities efficiently resonating at $\mathrm{THz}$ frequencies is challenging, due to the technological gap of $\mathrm{THz}$ materials and optical components, compared to other spectral regions. As concerns cavity mirrors, highly reflective dielectric coatings are scarcely developed at $\mathrm{THz}$ frequencies, and at present only, metallic coatings can be used, with a maximum reflectivity limited to $99.6 \%$ for gold. In addition, metallic mirrors are not suitable as input/ output couplers, because of unavoidable substrate absorption losses, and also the solutions commonly adopted at microwave frequencies (hole output coupling) have proven to be quite unsuitable for THz [144].

An alternative approach consists in using a wire-grid polarizer (WGP) as input/output coupler: if the electric field component is parallel to the metal wires, electrons are free to flow along the wires, and the incoming field experiences an almost complete reflection. However, a small amount of radiation leaks through the grid, allowing for the coupling of light in and out of the cavity. This approach has been adopted in a very few early experiments in far-IR spectroscopy [145-147], where finesses of about 14 and 3 were achieved at $690 \mathrm{GHz}$ and 1.5 THz frequencies, respectively (corresponding to Q-factors of about 7000 and 1500 , respectively). Braakman et al. demonstrated that, in the sub-THz range (around $300 \mathrm{GHz}$ ), resonant cavities based on WGPs can achieve Q-factors as large as $10^{5}$ [148], sufficient for a relevant enhancement. More recently, in the group led by Paolo De Natale, resonant cavities with different setups, specifically a V-shaped and a ring-shaped cavity, have been developed, and have been coupled to a THz QCL.Q-factors of about $2.5 \times 10^{5}$ have been reached, see Fig. 6 and [149]. At the same time, these cavities have been for the first time used to influence the QCL emission, paving the way to cavity linewidth narrowing. Finally, another cavity setup has been developed by the same group, which has reached for the first time an unprecedented Q-factor of $2.5 \times 10^{6}$, based on a bow tie design (Campa et al., submitted for publication).

\subsection{Cold Molecules}

Another intriguing playground, which could revolutionize $\mathrm{THz}$ frequency metrology, is the application of the above mentioned coherent sources to cold molecules, in analogy to what is currently happening in the mid-IR regime.

The shot-noise limit in a frequency measurement is given by $\delta \nu=1 / \tau N$, where $\tau$ is the coherence time for the measurement and $N$ is the number of detected molecules. The advantage offered by using a cold sample of molecule is twofold. First, by cooling the internal degrees of freedom of a molecular sample, the distribution of populated quantum states is 

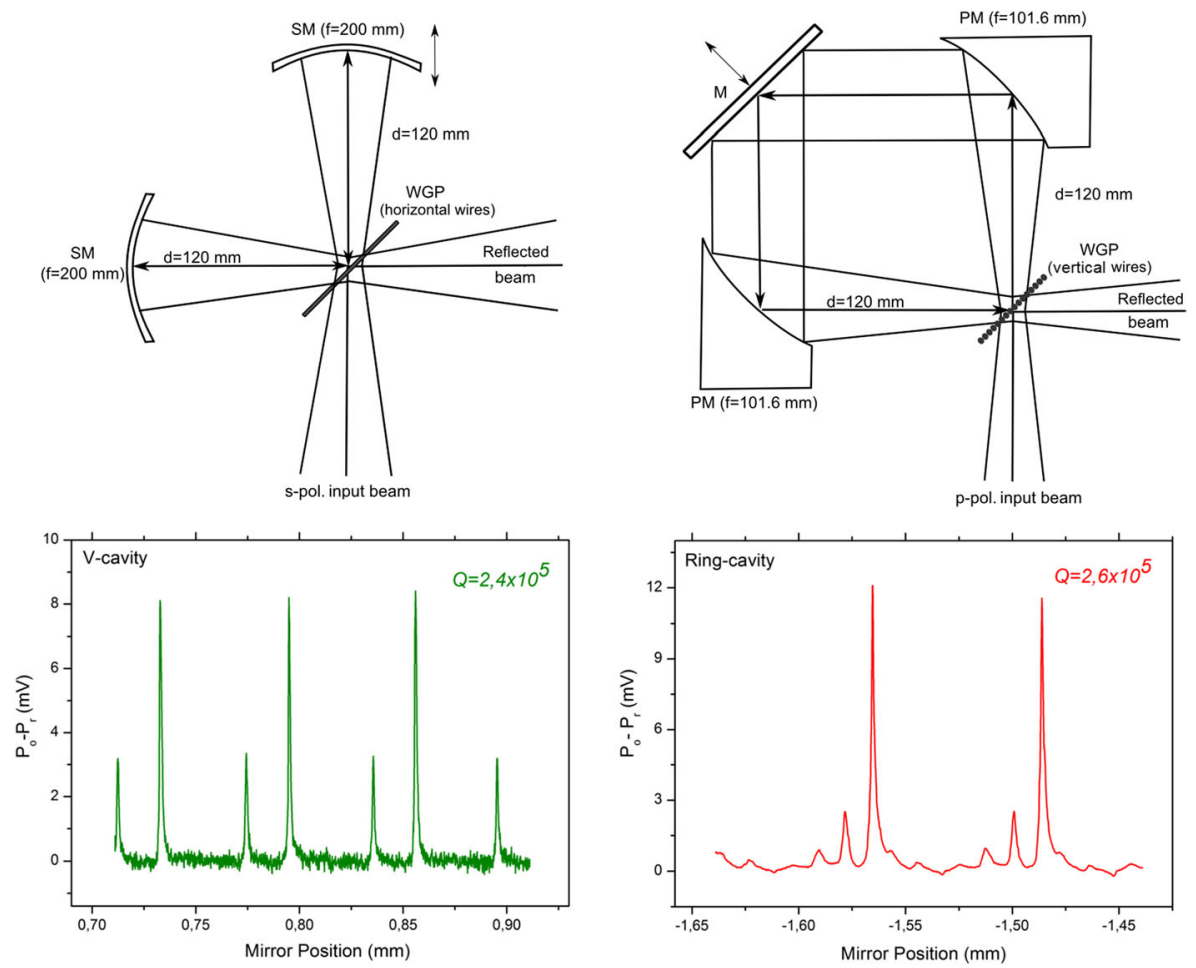

Fig. 6 Two different THz resonators setup and acquisitions: V-shaped cavity on the left with $2.4 \times 10^{5}$ achieved quality factor and ring-shaped setup on the right, with $2.6 \times 10^{5}$ achieved quality factor. Reprinted with permission from [149], copyright 2015

narrowed according to the Boltzmann statistics. Thus, $N$ is greatly enhanced if the state under investigation is the ground state, or a state that is energetically close to the ground state, compared with $\mathrm{k}_{\mathrm{B}} \mathrm{T}$. It is worth noticing that a present limit in molecular spectroscopy is posed by the lack of generally applicable methods to prepare a large population of molecules in highly excited states. Second, cooling the external degrees of freedom yields slower molecules that allow for measurements with longer coherence time $\tau$. Moreover, lower velocities yield reduced Doppler broadenings [150].

The coldest molecular species attainable to date are the ultracold alkali dimers created by associating ultracold atoms [151]. They have been employed, for instance, in the studies of ultracold bimolecular reactions $[152,153]$ and have been trapped in optical lattices to analyze their quantum dynamics, which represents the first step toward using these systems to explore many-body dynamics in regimes that are inaccessible to current theoretical techniques [154]. However, as interesting as these species are in the quest for new physical phenomena, they have not been at the focus of the spectroscopists' attention. Instead, their attention has been directed at less exotic molecular species, cooled by direct methods.

A conceptually simple method for preparing cold molecules is buffer-gas cooling, pioneered by J. Doyle [155]. Cooling is achieved via collisions with cryogenically cooled helium atoms, and the temperature of the cold molecules is typically around $1 \mathrm{~K}$. A large variety of atoms and molecules has been cooled using this technique, ranging from atoms and dimers to benzonitrile, fluorobenzene, anisole, for instance [156]. Further, buffer-gas cooling 
has been used for the production of molecular beams both of gaseous precursors and of laserablated species [157]. If curved guides are coupled to this kind of sources, the subset of molecules that are moving sufficiently slowly are extracted from the output of the source [158]. Spectroscopy on buffer-gas cooled species has been performed both inside the cooling cell $[156,159,160]$ and on the molecular beam [161]. Only very recently, cavity ringdown detection has been successfully used in a beam of buffer-gas cooled of acetylene [162].

Supersonic molecular beams are a classical method to produce cold molecular samples [163]. A molecular beam is generated by letting a gas expand from a high-pressure source into a low-pressure ambient background via a nozzle. Basically, a supersonic molecular beam is a fast-moving beam of internally cold molecules and it facilitates spectroscopic studies by providing molecules in an almost collision-free environment, thus with a strong reduction of inhomogeneous broadening. However, typical molecular beams have speeds of the order of $300-1000 \mathrm{~m} / \mathrm{s}$ and several techniques have been developed to manipulate and control their motional degrees of freedom.

Stark and Zeeman effects are common methods to manipulate polar and paramagnetic molecules with electric or magnetic fields, respectively [164-166]. In Stark decelerators, molecules with a permanent electric dipole moment (EDM) convert part of their kinetic energy into Stark energy upon entering an electric field, if they are in an appropriate quantum state. If the electric field is switched off before the molecule has left the electric field, the lost kinetic energy will not be returned. This process can be repeated over multiple stages until the molecules reach the desired final velocity. Once the average velocity is low enough, molecules can be loaded in a trap [167], for instance. Zeeman deceleration is entirely analogous, except that the force is exerted by a magnetic field on a magnetic dipole moment [168]. As a viable alternative to abruptly switching between different static field configurations, molecules can be captured in traveling potential wells $[169,170]$, directly from the supersonic molecular beam, and then decelerated. These methods are used to prepare a molecular beam in a single quantum state and at a mean speed typically adjustable between 400 and $500 \mathrm{~m} / \mathrm{s}$ to rest, with a translational temperature tunable from $1 \mathrm{~K}$ to $5 \mathrm{mK}$. High-resolution mid-IR spectroscopy of decelerated species has been performed for NH3 [171] and hydroxyl radicals (OH) [172, 173].

In these experiments, an interaction time as long as $1 \mathrm{~ms}$ was obtained. In 2008, Stuhl et al. [174] identified a class of diatomic molecules that presented almost-cycling transitions, which can be used for laser cooling. A couple of years later, DeMille group demonstrated the action of a radiative force acting on strontium monofluoride (SrF) [175] and then transverse laser cooling [176] and deceleration [177] of a SrF beam. Finally, in 2014, they were able to trap SrF in a threedimensional magneto-optical trap [178]. In the meanwhile, laser cooling of YO [179] and CaF [180] has also been reported. Typical temperatures reached with all these techniques are in the range of a half to about $100 \mathrm{mK}$, whereas the densities are in the range of $10^{7}$ molecules per $\mathrm{cm}^{3}$. Applying high-precision $\mathrm{THz}$ spectroscopy techniques to these cold molecule sources could in principle lead to an actual improvement for accuracy of at least two orders of magnitude.

\subsection{Frequency Referencing Improvement}

As previously discussed, the frequency standard in the $\mathrm{THz}$ region relies on Global Positioning System (GPS) dissemination of frequency standards, which is ubiquitous at present, providing the most widespread time and frequency reference for the majority of industrial and research applications worldwide. On the other hand, the ultimate limits of GPS presently hinder further 
advances in high-precision, scientific, and industrial applications relying on this dissemination scheme. This is not yet the case of $\mathrm{THz}$ technologies, as the actual frequency accuracy limit of $10^{-9}$ is not yet comparable with the accuracy given by GPS clock, but nevertheless far more accurate standards will become crucial in the next future. A timely exploitation of the continuous advances in the realization of new frequency standards is inherently tied to the uncertainty with which the standard is delivered from metrological institutes to the end users. Presently, GPS allows for traceability to the second in the International System of Units (SI) with a typical fractional frequency uncertainty of $10^{-13}$ on a day average [181]. This remarkable result can be obtained with a very simple equipment, consisting only of a receiver without any feedback from the user to the reference clock infrastructure. However, GPS dissemination severely limits the accuracy of current frequency standards and, even more, that of new optical atomic clocks [182], which have already shown fractional accuracy capabilities of $10^{-18}$ in a few hours of measurement [183-185]. This is the main reason why the development and characterization of long-haul optical fiber links (OFLs), rather than microwave satellite links, recently featured a substantial thrust [186-190]. Indeed, in the last years, OFLs have shown undoubted effectiveness in comparing remote atomic clocks beyond the ultimate limit of GPS-based comparisons, attaining precision levels not limited by the frequency transfer method [191-196], and are also envisioned as key elements for the development of a new kind of high-accuracy relativistic geodesy [195, 197]. Besides optical clock comparisons, short-range OFLs have recently been exploited in order to increase the spectral performance of a local laser and to allow for precision molecular spectroscopy [198]. It has been recently demonstrated that long-haul fiber-based optical frequency dissemination is a reliable tool for remote end users to perform high-precision procedures well beyond the ultimate capabilities of GPS dissemination [199]. The present dissemination of the fiber link in Italy is shown in Fig. 7.

\subsection{Novel QCL Sources}

Single-mode THz QCLs have already shown their huge potential for metrological grade $\mathrm{THz}$ spectroscopy [126] but, at the same time, QCL technology is making continuous leaps forward, toward new generation sources. One of the main objectives is to build THz QCLs able to operate at room temperature. In this regard, a very promising kind of source is based on intracavity difference frequency generation between two mid-IR QCLs [200]. These sources are currently the only semiconductor sources that are able to emit multi-mW power and cover the entire 1-5 THz range at room temperature [201, 202]. Wide-range frequency tuning spanning several THzs at room temperature based on composite distributed feedback (DFB) QCL arrays [203, 204], or external cavity technique [205], has been demonstrated. Monolithic electrical tuning is more convenient for practical applications. Monolithic tuning range of 3.44 to 4.02 THz was achieved with a dual-section DFG QCL waveguide design [206], and was further expanded to 2.6 to $4.2 \mathrm{THz}$ with a three-section DFG QCL waveguide design [207]. Very recently, by utilizing a low-loss buried-ridge waveguide design and highly dissipative epi-down mounting scheme, room temperature $\mathrm{CW}$ operation at $3.6 \mathrm{THz}$ was demonstrated with a continuous power of $3 \mu \mathrm{W}$ [208]. However, the relatively high threshold current density and low wall-plug efficiency (WPE) of the demonstrated devices prevented the room temperature $\mathrm{CW}$ operation of the monolithic tunable devices, which has been only recently demonstrated [209 and therein refs]. A careful characterization of these devices, regarding both their tunability characteristics and their spectral purity has been very recently performed by 


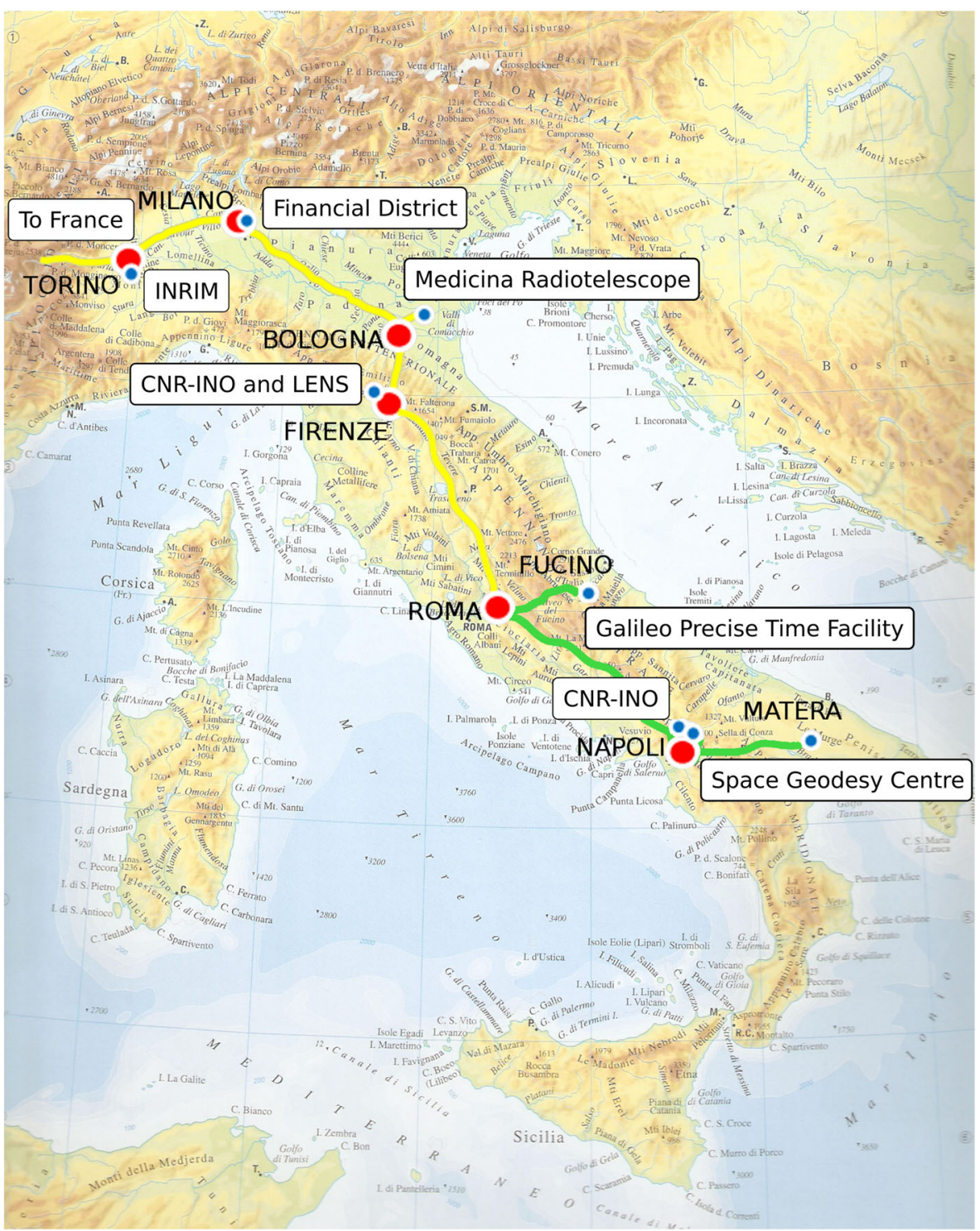

Fig. 7 Present day dissemination of the fiber link in Italy, in yellow the already working link, and in green the upcoming connections

(Consolino et al., submitted for publication), showing that not only these devices are able to work at room temperature, but that they are also suitable for metrological grade applications.

Another interesting and promising technology for THz high-precision spectroscopy is continuous-wave multimode broadband QCLs, whose operation has only recently been demonstrated [210, 211]. A broadband gain medium is desirable for a wide range of applications and, in QCL comb formation, is driven by ultrafast non-linearities (four-wave mixing) in the active region itself [212]. At terahertz frequencies, comb operation is also favored by the longer lifetimes of the optical transition upper state at those frequencies [213-215]. The ultrabroad 
gain bandwidth is achieved by fully exploiting the quantum engineering of intersubband transitions, integrating in the same laser ridge (resonator) different designs of a quantum cascade structure [216], tailored for different frequencies. First demonstrated in mid-IR QCLs [217], this heterogeneous cascade concept has also been successfully implemented in terahertz QCLs [218-220]. These sources show a comb emission regime with hundreds GHz spectral coverage, but their comb-like features and controllability have not been characterized, yet.

Open Access This article is distributed under the terms of the Creative Commons Attribution 4.0 International License (http://creativecommons.org/licenses/by/4.0/), which permits unrestricted use, distribution, and reproduction in any medium, provided you give appropriate credit to the original author(s) and the source, provide a link to the Creative Commons license, and indicate if changes were made.

\section{References}

1. F.C. De Lucia, The submillimeter: A spectroscopist's view, Journal of Molecular Spectroscopy 261, 1-17 (2010)

2. L. Testi, M. Zwaan, C. Vlahakis, S. Corder, Science verification datasets on the ALMA science portal Messenger 15017 (2012)

3. D. Leisawitz, W. Danchi, M. DjPjrro, et al., Scientific motivation andtechnology requirements for the SPIRIT and SPECS far-infrared/submillimeter space interferometers, Proc. SPIE, 4013 36-46 (2000)

4. M. Bellini, P. De Natale, G. Di Lonardo, L. Fusina, M. Inguscio, M. Prevedelli, Tunable far infrared spectroscopy of ${ }^{16} \mathrm{O}_{3}$ ozone, J. Mol. Spectros. 152 256-9 (1992)

5. N. C. Luhmann, W. A. Peebles, Instrumentation for magnetically confined fusion plasma diagnostics, Rev. Sci. Instrum. 55 279-331 (1984)

6. B. Gorshunov, A. Volkov, I. Spektor, A. Prokhorov, A. Mukhin, M. Dressel, S. Uchida, A. Loidl, Terahertz BWO-Spectrosopy, International Journal of Infrared and Millimeter Waves, 26 1217-1240 (2005)

7. G. Klapper, F. Lewen, R. Gendriesch, S. P. Belov, G. Winnewisser, Sub-Doppler Measurements of the Rotational Spectrum of ${ }^{13} \mathrm{C}^{16} \mathrm{O}$, J. Mol. Spectrosc. 201, 124-127 (2000)

8. R. Köhler, A. Tredicucci, F. Beltram, H. E. Beere, E. H. Linfield, A. G. Davies, D. A. Ritchie, R. C. Iotti, F. Rossi, Terahertz semiconductor-heterostructure laser, Nature 417 156-9 (2002)

9. S. Fathololoumi, E. Dupont, C. W. Chan, Z. R. Wasilewski, S. R. Laframboise, D. Ban, A. Mátyás, C. Jirauschek, Q. Hu, H. C. Liu, Terahertz quantum cascade lasers operating up to $\sim 200 \mathrm{~K}$ with optimized oscillator strength and improved injection tunneling, Opt. Express 203866 (2012)

10. M. S. Vitiello and A. Tredicucci, Tunable Emission in THz Quantum Cascade Lasers, IEEE Trans. Terahertz Sci. Technol. 1 76-84 (2011)

11. R. F. Kazarinov, R. A. Suris, Possible amplification of electromagnetic waves in a semiconductor with a superlattice, Sov. Phys. Semicond. 5 707-9 (1971)

12. Q. Qin, B.S. Williams, S. Kumar, J.L. Reno, Q. Hu, Tuning a terahertz wire laser, Nature Photon. 3 732-7 (2009)

13. J.M. Reix, T. Passvogel, G. Crone, B. Collaudin, P. Rideau, Y. Roche, C. Vogel, The Herschel/Planck programme, technical challenges for two science missions, successfully launched, Acta Astron. 66 130-48 (2010)

14. D.M. Mittleman, Sensing with THz Radiation (Berlin: Springer) (2003)

15. M. Tonouchi, Cutting-edge terahertz technology, Nature Photon. 1 97-105 (2007)

16. F. Capasso, R. Paiella, Quantum cascade lasers: ultrahigh-speed operation, optical wireless communication, narrow linewidth, and far-infrared emission, IEEE J. Quantum Electron. 38 511-32 (2002)

17. S. Bartalini, S. Borri, P. Cancio, A. Castrillo, I. Galli, G. Giusfredi, D. Mazzotti, L. Gianfrani, P. De Natale, Observing the intrinsic linewidth of a quantum-cascade laser: beyond the Schawlow-Townes limit, Phys Rev. Lett. 104 1-4 (2010)

18. M.S. Vitiello, L. Consolino, S. Bartalini, A. Taschin, A. Tredicucci, M. Inguscio, P. De Natale, Quantumlimited frequency fluctuations in a terahertz laser, Nature Photon. 6 525-8 (2012)

19. M. Ravaro, S. Barbieri, G. Santarelli, V. Jagtap, C. Manquest, et al. Measurement of the intrinsic linewidth of terahertz quantum cascade lasers using a near-infrared frequency comb, Optics Express, 20 (23), pp.25654-25661 (2012)

20. A.L. Betz, R.T. Boreiko, B.S. Williams, S. Kumar, Q. Hu, J.L. Reno, Frequency and phase-lock control of a $3 \mathrm{THz}$ quantum cascade laser, Opt Lett. 30 1837-9 (2005) 
21. A.A. Danylov,T.M. Goyette, J. Waldman, M.J. Coulombe, A.J. Gatesman, R.H. Giles, W.D. Goodhue, X. Qian, W.E. Nixon, Frequency stabilization of a single mode terahertz quantum cascade laser to the kilohertz level, Opt. Express 17 7525-32 (2009)

22. d. Rabanus, U.U. Graf, M. Philipp,O. Ricken, J. Stutzki, B. Vowinkel, M.C. Wiedner, C. Walther, M. Fischer, J. Faist, Phase locking of a 1.5 Terahertz quantum cascade laser and use as a local oscillator in a heterodyne HEB receiver, Opt. Express 17 1159-68 (2009)

23. P. Khosropanah, A. Baryshev, W. Zhang, W. Jellema, J.N. Hovenier, J.R. Gao, T.M. Klapwijk, D.G. Paveliev, B.S. Williams, S. Kumar, Q. Hu, J.L. Reno, B. Klein, J.L. Hesler, Phase locking of a $2.7 \mathrm{THz}$ quantum cascade laser to a microwave reference, Opt. Lett. 34 2958-60 (2009)

24. A.A. Danylov, A.R. Light, J. Waldman, N.R. Erickson, X. Qian, W.D. Goodhue, 2.32 THz quantum cascade laser frequency-locked to the harmonic of a microwave synthesizer source, Opt. Express 20 27908-14 (2012)

25. H.W. Hübers, R. Eichholz, S.G. Pavlov, H. Richter, High resolution terahertz spectroscopy with quantum cascade lasers, J. Infrared Millim. Terahertz Waves 34 325-41 (2013)

26. H. Richter, S.G. Pavlov, A.D. Semenov, L. Mahler, A. Tredicucci, H.E. Beere, D.A. Ritchie, H.W. Hübers, Submegahertz frequency stabilization of a terahertz quantum cascade laser to a molecular absorption line, Appl. Phys. Lett. 9671112 (2010)

27. Y. Ren, J.N. Hovenier, M. Cui, D.J. Hayton, J.R. Gao, T.M. Klapwijk, S.C. Shi, T.Y. Kao, Q. Hu, J.L. Reno, Frequency locking of single-mode 3.5-THz quantum cascade lasers using a gas cell, Appl. Phys. Lett. $100041111(2012)$

28. S. Barbieri, P. Gellie, G. Santarelli, L. Ding, W. Maineult, C. Sirtori, R. Colombelli, H.E. Beere, D.A. Ritchie, Phase-locking of a 2.7-THz quantum cascade laser to a mode-locked erbium-doped fibre laser, Nature Photon. 4 636-40 (2010)

29. T. Löffler, T. May, C. am Weg, A. Alcin, B. Hils, H.G. Roskos, Continuous-wave terahertz imaging with a hybrid system, Appl. Phys. Lett. 90 91111(2007)

30. M. Ravaro, C. Manquest, C. Sirtori, S. Barbieri, G. Santarelli, K. Blary, J.F. Lampin, S.P. Khanna, E.H. Linfield, Phase-locking of a $2.5 \mathrm{THz}$ quantum cascade laser to a frequency comb using a GaAs photomixer, Opt. Lett. 36 3969-71 (2011)

31. T. Yasui, S. Yokoyama, H. Inaba, K. Minoshima, T. Nagatsuma, T. Araki, Terahertz frequency metrology based on frequency comb, IEEE J. Sel. Top. Quantum Electron. 17 191-201 (2011)

32. G. Klatt, R. Gebs, C. Janke, T. Dekorsy, A. Bartels ,Rapid-scanning terahertz precision spectrometer with more than $6 \mathrm{THz}$ spectral coverage, Opt. Express 17 22847-54 (2009)

33. L. Consolino, A. Taschin, P. Bartolini, S. Bartalini, P. Cancio, A. Tredicucci, H. E. Beere, D. A. Ritchie, R. Torre, M. S. Vitiello, P. De Natale, Phase-locking to a free-space terahertz comb for metrological-grade terahertz lasers, Nature Commun. 31040 (2012)

34. G.A. Askaryan, Cherenkov and transition radiation from electromagnetic waves, Sov. Phys. JETP 42 1360-4 (1962)

35. S.B. Bodrov, A.N. Stepanov, M.I. Bakunov, B.V. Shishkin, I.E. Ilyakov, R.A. Akhmedzhanov, Highly efficient optical-to-terahertz conversion in a sandwich structure with $\mathrm{LiNbO}_{3}$ core, Opt. Express 171871 (2009)

36. A. Maestrini, J. Ward, J. Gill, H. Javadi, E. Schlecht, G. Chattopadhyay, F. Maiwald, N.R. Erickson, and I. Mehdi, A 1.7 to $1.9 \mathrm{THz}$ Local Oscillator Source, IEEE Microwave and Wireless Components Letters, 14 253-255 (2004)

37. J. Ward, E. Schlecht, G. Chattopadhyay, H. Javadi, J. Gill and I. Mehdi \& A. Maestrini, C. TriponCanseliet, Local Oscillators from 1.4 to $1.9 \mathrm{THz}$, Proceedings of the 16th International Symposium on Space Terahertz Technology, Göteborg, Sweden (2005)

38. A. Maestrini, Frequency Multipliers for Local Oscillators at THz Frequencies, Proceedings of the 4th ESA Workshop on Millimetre Wave Technology and Applications, Espoo, Finland (2006)

39. C.-Y.E Tong, D. Meledin, D. Loudkov, R. Blundell, N. Erickson, J. Kawamura, I. Mehdi, G. Gol'tsman, A $1.5 \mathrm{THz}$ Hot-Electron Bolometer mixer operated by a planar diode based local oscillator, Proc. IEEE MTTS International, Vol. 2, pp. 751-754, Philadelphia, Pennsylvania (2003)

40. D. Marrone, R. Blundell, E. Tong, S. Paine, D. Loudkov, J. Kawamura, D. Luhr, C. Barrientos, Observations in the 1.3 and $1.5 \mathrm{THz}$ atmospheric windows with the Receiver Lab Telescope, Proceedings of the Sixteenth International Symposium on Space Terahertz Technology, Göteborg, Sweden (2005)

41. J.T. Louhi, A.V. Räisänen, N.R. Erickson, Cooled Schottky varactor frequency multipliers at submillimeter wavelengths, IEEE Trans. Microwave Theory Tech., 41 565-571 (1993)

42. A. Maestrini, J. S. Ward, H. Javadi, C. Tripon-Canseliet, J. Gill, G. Chattopadhyay, E. Schlecht, and I. Mehdi, Local Oscillator Chain for 1.55 to $1.75 \mathrm{THz}$ with $100 \mu \mathrm{W}$ Peak Power, IEEE Microwave and Wireless Components Letters, 15 871-873 (2005) 
43. A.V. Räisänen, Frequency multipliers for millimeter and submillimeter wavelengths, Proc. IEEE, 8 18421852 (1992)

44. T. Crowe, R. Zimmermann, Progress Toward Solid State Local Oscillators at $1 \mathrm{THz}$, IEEE Microwave and Guided Wave Letters, 65 207-208(1996)

45. P. Zimmerman, Multipliers for terahertz local oscillators, Proc. SPIE: Advanced Technology MMW, Radio, and Terahertz Telescopes, 3357 152-158 (1998)

46. N.J. Cronin, V.J. Law, Planar Millimeter-Wave Diode Mixer, IEEE Trans. Microwave Theory Tech, 33 827-830 (1985)

47. W.L. Bishop, K. McKinney, R.J. Mattauch, T.W. Crowe, and G. Green, A novel whiskerless Schottky diode for millimeter and submillimeter wave applications, 1987 I.E. MTT-S Int. Microwave Symp. Dig., Vol. II, 607-610 (1987)

48. N.R. Erickson, High efficiency submillimeter frequency multipliers, Proc. IEEE MTT-S International, 1301-1304 (1990)

49. N.R. Erickson, B.J. Rizzi, A High Power Doubler for $174 \mathrm{GHz}$ Using a Planar Diode Array, Proc. 4th International Symposium on Space Terahertz Technology, 287-295 (1993)

50. B.J. Rizzi, T. Crowe, N. R Erickson, A high-power millimeter-wave frequency doubler using a planar diode array, IEEE Microwave and Guided Wave Letters, 3 188-190 (1993)

51. A. Rydberg, H. Grönqvist, E. Kollberg, Millimeter- and Submillimeter-Wave Multipliers using Quantum Barrier-Varactor (QBV) Diodes, IEEE Electron Device Letters, 11 373-375 (1990)

52. X. Mélique, A. Maestrini, P. Mounaix, M. Favreau, G. Beaudin, G. Goutoule, T. Närhi and D. Lippens, Fabrication and performance of InP-based Heterostructure Barrier Varactors in a $250 \mathrm{GHz}$ Waveguide Tripler, IEEE Trans. Microwave Theory Tech., 48 1000-1006 (2000)

53. M. Krach, J. Freyer, M. Claassen, An integrated ASV frequency tripler for millimeter-wave applications, Proc. 33rd European Microwave Conference, 3 1279-1281 (2003)

54. J. Tuovinen, N.R. Erickson, Analysis of a $170 \mathrm{GHz}$ frequency doubler with an array of planar diodes, IEEE Trans. Microwave Theory Tech., 43 962-968 (1995)

55. B.J. Drouin, F.W. Maiwald, J.C. Pearson, Application of cascaded frequency multiplication to molecular spectroscopy, Rev. Sci. Instrum., 76093113 (2005)

56. J.C. Pearson, B.J. Drouin, A. Maestrini, I. Mehdi, J. Ward, R.H. Lin, et al., Demonstration of a room temperature 2.48-2.75 THz coherent spectroscopy source, Rev. Sci. Instrum. 82093105 (2011)

57. B.J. Drouin, Isotopic Spectra of the Hydroxyl Radical, J. Phys. Chem. A, 117 10076-10091 (2013)

58. B.J. Drouin, J.P. Pearson, S. Yu, H. Gupta, Characterization and Use of a 1.3-1.5 THz Multiplier Chain for Molecular Spectroscopy, IEEE Trans. Terahertz Sci. Technol., 3 314-321 (2013)

59. D.D. Bicanic, B.F.J. Zuidberg and A. Dymanus, Generation of continuously tunable laser sidebands in the submillimeter region, Appl. Phys. Lett. 32, 367-369 (1978)

60. W.A.M. Blumberg, H.R. Fettermand, D. Peck and P.F. Goldsmith, Tunable submillimeter sources applied to the excited state rotational spectroscopy and kinetics of $\mathrm{CH}_{3} \mathrm{~F}$, Appl. Phys. Lett. 35, 582-585 (1979)

61. J. Farhoomand, G.A. Blake, M.A. Frerking and H.M. Pickett Generation of tunable laser sidebands in the far-infrared region J. Appl. Phys. 57, 1763-1766 (1985)

62. G. Piau, F.X. Brown, D. Dangoisse and P. Glorieux Heterodyne detection of tunable FIR sidebands. IEEE J. Quantum Electron. QE-23, 1388-1391 (1987)

63. K.M. Evenson, D.A. Jennings and F.R. Petersen Tunable far-infrared spectroscopy. Appl. Phys. Lett. 44, 576-578 (1984)

64. L.R. Zink, P. De Natale, F.S. Pavone, M. Prevedelli, K.M. Evenson and M. Inguscio Rotational far infrared spectrum of ${ }^{13}$ CO, Journal of Molecular Spectroscopy 143, 304-310 (1990)

65. I.G. Nolt, J.V. Radostitz, G. Di Lonardo, K.M. Evenson, D.A. Jennings, K.R. Leopold, M.D. Vanek, L.R. Zink, A. Hinz and K.V. Chance Accurate rotational constants of CO, HCl, and HF: Spectral standards for the 0.3 - to 6-THz (10- to 200- $\left.\mathrm{cm}^{-1}\right)$ region J. Mol. Spectrosc. 125, 274-287 (1987)

66. S. Viciani and F. Marin P. De Natale Noise characterization of a coherent tunable far infrared spectrometer Review of Scientific Instruments 69, 372 (1998);

67. E. R. Brown, F. W. Smith, and K. A. McIntosh, Coherent millimeter-wave generation by heterodyne conversion in low-temperature-grown GaAs photoconductors, J. Appl. Phys. 73, 1480 (1993).

68. S. Verghese, K. A. McIntosh, and E. R. Brown, Highly tunable fiber-coupled photomixers with coherent terahertz output power, IEEE Trans. Microwave Theory and Tech. 45, 1301 (1997).

69. S. Matsuura, M. Tani, and K. Sakai, Generation of coherent terahertz radiation by photomixing in dipole photoconductive antennas, Appl. Phys. Lett. 70, 559 (1997).

70. M. Kominami, D. M. Pozar, and D. H. Schaubert, Dipole and Slot Elements and Arrays on Semi-Infinite Substrates,IEEE Trans. Antennas Propag. 33, 600 (1985). 
71. S. M. Duffy, S. Verghese, K. A. McIntosh, A. Jackson, A. C. Gossard, and S. Matsuura, Accurate modeling of dual dipole and slot elements used with photomixers for coherent terahertz output power, IEEE Trans. Microwave Theory and Tech. 49, 1032 (2001).

72. S. Matsuura and H. Ito, in Terahertz Optoelectronics, edited by K. Sakai, Springer, Berlin, pp. 173-179 (2005)

73. S. Matsuura and H. Ito, in Terahertz Optoelectronics, edited by K. Sakai, Springer, Berlin, pp. 179-186 (2005)

74. K.H. Yang, P.L. Richards, Y.R. Shen, Generation of far-infrared radiation by picosecond light pulses in $\mathrm{LiNbO}_{3}$, Appl. Phys. Lett., 19, 320 (1971)

75. X.C. Zhang,Y. Jin,X.F. Ma, Coherent measurement of terahertz optical rectification from electrooptic crystals, Appl. Phys. Lett., 61, 2764 (1992)

76. B.B. Hu, X.C. Zhang,D.H. Auston, Free-space radiation from electrooptic crystals, Appl. Phys. Lett., 56, 506 (1990)

77. A. Nahata, A.S. Weling,T.F. Heinz, A wideband coherent terahertz spectroscopy system using optical rectification and electrooptic sampling, Appl. Phys. Lett., 69, 2321 (1996)

78. P.Y. Han, X.C. Zhang, Free-space coherent broadband terahertz time-domain spectroscopy, Meas. Sci. Technol., 12, 1747 (2001)

79. A. Rice, Y. Jin, X. F. Ma, X.-C. Zhang, Terahertz optical rectification from $<110>$ zinc-blende crystals, Appl. Phys. Lett., 64, 1324 (1994)

80. M. Nagai, K. Tanaka, Generation and detection of terahertz radiation by electrooptical process in GaAs using $1.56 \mu \mathrm{m}$ fiber laser pulses, Appl. Phys. Lett., 85, 3974 (2004)

81. R. Huber, A. Brodschelm, F. Tauser, and A. Leitenstorfer, Generation and field resolved detection of femtosecond electromagnetic pulses tunable up to 41 THz, Appl. Phys. Lett., 76, 3191 (2000)

82. W. Shi, Y. J. Ding, N. Fernelius, and K. Vodopyanov, Efficient, tunable and coherent $0.18-5.27-\mathrm{THz}$ source based on GaSe crystal, Opt. Lett., 27, 1454 (2002)

83. X. Xie, J. Xu, and X.C. Zhang, Terahertz wave generation and detection from a CdTe crystal characterized by different excitation wavelengths, Opt. Lett., 31, 978 (2006)

84. K. Liu, H.-S. Kang, T.-K. Kim, and X.-C. Zhang, Study of ZnCdTe crystals as THz wave emitters and detectors, Appl. Phys. Lett., 81, 4115 (2002)

85. Y. J. Ding, High-power tunable terahertz sources based on parametric processes and applications, IEEE J. Sel. Top. Quantum Electron. 13, 705-720 (2007).

86. M. Scheller, J. M. Yarborough, J. V. Moloney, M. Fallahi, M. Koch, and S. W. Koch, Room temperature continuous wave milliwatt terahertz source, Opt. Express 18, 27112-27117 (2010)

87. K. Takeya, T. Minami, H. Okano, S. R. Tripathi, and K. Kawase Enhanced Cherenkov phase matching terahertz wave generation via a magnesium oxide doped lithium niobate ridged waveguide crystal, APL Photonics 2, 016102 (2017)

88. K. Kawase, M. Mizuno, S. Sohma, H. Takahashi, T. Taniuchi, Y. Urata, S. Wada, H. Tashiro, and H. Ito, Difference-frequency terahertz-wave generation from 4-dimethylamino-N-methyl-4-stilbazolium-tosylate by use of an electronically tuned Ti:sapphire laser, Opt. Lett. 24 (15), 1065-1067 (1999)

89. K. Kawase, T. Hatanaka, H. Takahashi, K. Nakamura, T. Taniuchi, and H. Ito, Tunable terahertz-wave generation from DAST crystal by dual signal-wave parametric oscillation of periodically poled lithium niobate, Opt. Lett. 25 (23), 1714-1716 (2000)

90. T. Taniuchi, J. Shikata, and H. Ito, Tunable THz-wave generation in DAST crystal with dual-wavelength KTP optical parametric oscillator, Electron. Lett. 36 (16), 1414-1416 (2000)

91. H. Ito, K. Suizu, T. Yamashita, A. Nawahara, and T. Sato, Random frequency accessible broad tunable terahertz-wave source using phase-matched 4-dimethylamino-N-methyl-4-stilbazolium tosylate crystal, Jpn. J. Appl. Phys. 46 (11), 7321-7324 (2007)

92. K. Suizu, K. Miyamoto, T. Yamashita, and H. Ito, High-power terahertz-wave generation using DAST crystal and detection using mid-infrared powermeter, Opt. Lett. 32 (19), 2885-2887 (2007)

93. K. Miyamoto, H. Minamide, M. Fujiwara, H. Hashimoto, and H. Ito, Widely tunable terahertz-wave generation using an N-benzyl-2-methyl-4-nitroaniline crystal, Opt. Lett. 33 (3), 252-254 (2008)

94. K. Miyamoto, S. Ohno, M. Fujiwara, H. Minamide, H. Hashimoto, and H. Ito, Optimized terahertz-wave generation using BNA-DFG. Opt. Express 17, 14832-14838 (2009)

95. R. Sowade, I. Breunig, I. Camara Mayorga, J. Kiessling, C. Tulea, V. Dierolf, and K. Buse, Continuouswave optical parametric terahertz source, Opt. Express 17 22303-10 (2009)

96. j. Kiessling, F. Fuchs, K. Buse, and I. Breunig, Pump-enhanced optical parametric oscillator generating continuous wave tunable terahertz radiation, Opt. Lett. 36 4374-6 (2011)

97. J. Kiessling, I. Breunig, P. G. Schunemann, K. Buse and K. L. Vodopyanov, New Journal of Physics 15 105014 (2013) 
98. C. Freed and A. Javan, Standing-wave saturation resonances in the $\mathrm{CO}_{2}$ 10.6- $\mu$ transitions observed in a low-pressure room-temperature absorber gas, Appl. Phys. Lett. 17, 53-56 (1970)

99. F.R. Petersen, E.C. Beatty and C.R. Pollock Improved rovibrational constants and frequency tables for the normal laser bands of 12C 16O2. J. Mol. Spectrosc. 102, 112-122 (1983)

100. L. C. Bradley, K.L. Soohoo and C. Freed Absolute frequencies of lasing transitions in nine $\mathrm{CO} 2$ isotopic species. IEEE J. Quantum Electron. QE-22, 234-267 (1986)

101. F. Matsushima, H. Odashima, D. Wang, S. Tsunekawa and K. Takagi, Far-Infrared Spectroscopy of LiH using a Tunable Far-Infrared Spectrometer. Jpn. J. Appl. Phys. 33, 315-318 (1994)

102. L. Fusina, P. De Natale, M. Prevedelli and L.R. Zink The submillimeter rotation spectrum of DCl. J. Mol. Spectrosc. 152, 55 (1992)

103. G. Di Lonardo, L. Fusina, P. De Natale et al. The pure rotation spectrum of $\mathrm{HBr}$ in the submillimeter-wave region J. Mol. Spectrosc. 148, 86 (1991)

104. M. Bellini, P. De Natale, G. Di Lonardo et al. Tunable far infrared spectroscopy of $16 \mathrm{O}_{3}$ ozone. J. Mol. Spectrosc. 152, 256 (1992)

105. G. Di Lonardo, L. Fusina, M. Bellini, P. De Natale, G. Buffa and O. Tarrini Air-Broadening of Rotational Lines of Ozone in the 1.5-THz Region Journal of Molecular Spectroscopy 161, 581-584 (1993)

106. K. Chance, P. De Natale, M. Bellini, M. Inguscio, G. Di Lonardo and L. Fusina Pressure Broadening of the 2.4978-THz Rotational Lines of $\mathrm{HO}_{2}$ by $\mathrm{N}_{2}$ and $\mathrm{O}_{2}$, Journal of Molecular Spectroscopy 163, 67-70 (1994)

107. G. Cazzoli, L. Cludi, G. Cotti, L. Dore, C. Degli Esposti, M. Bellini and P. De Natale, The Rotational Spectrum of $\mathrm{CHF}_{3}$ in the Submillimeter-Wave and Far-Infrared Region: Observation of the $\mathrm{K}=3$ Line Splitting Journal of Molecular Spectroscopy 163, 521-528 (1994).

108. P. De Natale, M. Bellini, M. Inguscio, G. Buffa and O. Tarrini Far-Infrared Collisional Lineshapes of Lithium Hydride and Deuteride Perturbed by $\mathrm{H}_{2}$ and $\mathrm{D}_{2}$ Journal of Molecular Spectroscopy 163, 510-514 (1994)

109. M. Bellini, P. De Natale, M. Inguscio, E. Fink, D. Galli and F. Palla Laboratory measurements of rotational transitions of lithium hydride in the Far-Infrared Astrophysical Journal 424, 507-509 (1994)

110. M. Bellini, P. De Natale, L. Fusina and G. Modugno The Pure Rotation Spectrum of $\mathrm{HOCl}$ in the Submillimeter-Wave Region Journal of Molecular Spectroscopy 172, 559-562 (1995)

111. P. De Natale, L. Lorini, M. Inguscio, G. Di Lonardo, L. Fusina, P.A.R. Ade and A.G. Murray Improved sensitivity of tunable far-infrared spectroscopy: application to the detection of $\mathrm{HBr}$ in the $\mathrm{v}=1$ state Applied Optics 36, 5822-5826 (1997)

112. P. De Natale, L. Lorini, M. Inguscio, I.G. Nolt, J. Park, G. Di Lonardo, L. Fusina, P.A.R. Ade and A.G. Murray Accurate frequency measurements for $\mathrm{H}_{2} \mathrm{O}$ and ${ }^{16} \mathrm{O}_{3}$ in the $119-\mathrm{cm}^{-1} \mathrm{OH}$ atmospheric window Applied Optics, 36, 8526-8532 (1997)

113. P. De Natale, L. Lorini, M. Inguscio, G. Di Lonardo and L. Fusina High-sensitivity detection of the rotation spectrum of $\mathrm{HCl}$ in the $\mathrm{v}=1$ state by tunable FIR spectroscopy Chemical Physics Letters, 273, 253-258 (1997)

114. P. De Natale, M. Bellini, W. Goetz, M. Prevedelli, and M. Inguscio, Hyperfine structure and isotope shift in the far-infrared ground-state transitions of atomic oxygen. Phys. Rev. A 48, 3757 (1993)

115. M. Inguscio, P. De Natale and L. Veseth Isotopic shift in atomic fine structure theory and experiment for oxygen transitions in the far infrared Comments. At. Mol. Phys. 30, 3-13 (1994)

116. J.M. Brown, K.M. Evenson and L.R. Zink, Laser magnetic-resonance measurement of the ${ }^{3} \mathrm{P}_{1}-{ }^{3} \mathrm{P}_{2}$ finestructure splittings in ${ }^{17} \mathrm{O}$ and ${ }^{18} \mathrm{O}$, Phys. Rev. A 48, 3761-3763 (1993)

117. G. Modugno, P. De Natale, M. Bellini, M. Inguscio, G. Di Lonardo, L. Fusina, J. Vander Auwera, Precise measurement of molecular dipole moments with a tunable far-infrared Stark spectrometer: application to HOCl. J. Opt. Soc. Am. B 13, 1645 (1996)

118. M. Bellini, E. Catacchini, P. De Natale, G. Di Lonardo, L. Fusina, M. Inguscio, E. Venuti, Stark and Frequency Measurements in the FIR Spectrum of $\mathrm{H}_{2} \mathrm{O}_{2}$, Journal of Molecular Spectroscopy, 177 115-123 (1996)

119. S. Viciani, F. Marin, P. De Natale, Noise characterization of a coherent tunable far infrared spectrometer, Review Of Scientific Instruments 69, 372-376 (1998)

120. P. De Natale, L. Gianfrani, S. Viciani and M. Inguscio Spectroscopic observation of the Faraday effect in the far infrared. Opt. Lett. 22, 1896 (1997)

121. S. Viciani, P. De Natale, L. Gianfrani, and M. Inguscio, Magnetic-field effects on molecular transitions in the far-infrared region: prospects for more-sensitive spectrometers, J. Opt. Soc. Am. B 16, 301 (1999)

122. H. Odashima, L.R. Zink and K.M. Evenson Tunable far-infrared spectroscopy extended to $9.1 \mathrm{THz}$ Opt. Lett. 24, 406-407 (1999)

123. A. J. Deninger, A. Roggenbuck, S. Schindler, S. Preu, $2.75 \mathrm{THz}$ tuning with a triple-DFB laser system at $1550 \mathrm{~nm}$ and InGaAs photomixers J Infrared Milli Terahz Waves, 36 269-277 (2015) 
124. T. Sasaki, T. Tanabe, J.-I. Nishizawa, Frequency Stabilized GaP Continuous-Wave Terahertz Signal Generator for High-Resolution Spectroscopy, Optics and Photonics Journal, 4, 8-13 (2014)

125. H.-W. Hübers, S. G. Pavlov, H. Richter, A. D. Semenov, L. Mahler, A. Tredicucci, H. E. Beere, D. A. Ritchie, High-resolution gas phase spectroscopy with a distributed feedback terahertz quantum cascade laser, Appl. Phys. Lett. 89, 061115 (2006)

126. S. Bartalini, L. Consolino, P. Cancio, P. De Natale, P. Bartolini, A. Taschin, M. De Pas, H. Beere, D. Ritchie, M. S. Vitiello, and R. Torre, Frequency-Comb-Assisted Terahertz Quantum Cascade Laser Spectroscopy Phys. Rev. X 4, 021006 (2014)

127. L.D. Carr, D. DeMille, R.V. Krems, and J. Ye, Cold and ultracold molecules: science, technology and applications. New J. Phys. 11055049 (2009)

128. T. Steinmetz, T. Wilken, C. Araujo-Hauck, R. Holzwarth, T.W. Hänsch, L. Pasquini, A. Manescau, S. D'Odorico, M.T. Murphy, T. Kentischer, W. Schmidt, T. Udem, Laser frequency combs for astronomical observations, Science 5321 1335-7 (2008)

129. G. Schettino, E. Oliva, M. Inguscio, C. Baffa, E. Giani, A. Tozzi, and P. Cancio, Optical frequency comb as a general-purpose and wide-band calibration source for astronomical high resolution infrared spectrographs, Exp. Astron. 3169 (2011)

130. M.S. Vitiello, L. Viti, L. Romeo, D. Ercolani, G. Scalari, J. Faist, F. Beltram, L. Sorba, and A. Tredicucci, Semiconductor nanowires for highly sensitive, room-temperature detection of terahertz quantum cascade laser emission, Appl. Phys. Lett. 100241101 (2012)

131. M.S. Vitiello, D. Coquillat, L. Viti, D. Ercolani, F. Teppe, A. Pitanti, F. Beltram, L. Sorba, W. Knap and A. Tredicucci, Room-temperature terahertz detectors based on semiconductor nanowire field-effect transistors, Nano Lett. 12 96-101 (2012)

132. L. Vicarelli, M.S. Vitiello, D. Coquillat, A. Lombardo, A.C. Ferrari, W. Knap, M. Polini, V. Pellegrini and A. Tredicucci, Graphene field-effect transistors as room-temperature terahertz detectors Nature Mater. 11 865-71 (2012)

133. C.C. Costain, The use of saturation dip absorption in microwave spectroscopy and in microwave frequency stabilization. Can. J. Phys., 47, 2431-2433 (1969)

134. R.S. Winton, W. Gordy, High precision millimeter-wave spectroscopy with the Lamb dip. Phys. Lett. A, 32, 219-220 (1979)

135. G. Cazzoli and C. Puzzarini, Sub-Doppler Resolution in the THz Frequency Domain: $1 \mathrm{kHz}$ Accuracy at 1 THz by Exploiting the Lamb-Dip Technique. J. Phys. Chem. A, 117, 13759-13766 (2013)

136. T.L. Nicholson, S.L. Campbell, R.B. Hutson, G.E. Marti, B.J. Bloom, R.L. McNally, W. Zhang, M.D. Barrett, M.S. Safronova, G.F. Strouse, W.L. Tew and J. Ye, Systematic evaluation of an atomic clock at $2 \times 10^{-18}$ total uncertainty, Nature Comm. 66896 (2015)

137. S. Borri and G. Santambrogio, Laser spectroscopy of cold molecules,Advances in Physics: X, 1 Iss. 3 (2016)

138. S. Haroche, "Nobel Lecture: Controlling photons in a box and exploring the quantum to classical boundary," Rev. Mod. Phys. 85, 1083-1102 (2013).

139. G. Gagliardi, H.-P. Loock (eds.), Cavity-Enhanced Spectroscopy and Sensing, Springer Series in Optical Sciences 179, DOI 10.1007/978-3-642-40003-2_1, Springer-Verlag Berlin Heidelberg (2014)

140. S. Kuhr, S. Gleyzes, C. Guerlin, J. Bernu, U. B. Hoff, S. Deléglise, S. Osnaghi, M. Brune, J.-M. Raimond, S. Haroche, E. Jacques, P. Bosland, and B. Visentin, "Ultrahigh finesse Fabry-Pérot superconducting resonator," Appl. Phys. Lett. 90, 164101 (2007).

141. G. A. Gary, E. A. West, D. Rees, J. A. McKay, M. Zukic, and P. Herman, "Solar CIV vacuum-ultraviolet Fabry-Pérot interferometers," Astron. Astrophys. 461, 707-722 (2007).

142. G. Rempe, R. J. Thompson, H. J. Kimble, and R. Lalezari, "Measurement of ultralow losses in an optical interferometer," Opt. Lett. 17, 363-365 (1992).

143. A. A. Savchenkov, A. B. Matsko, V. S. Ilchenko, and L. Maleki, "Optical resonators with ten million finesse," Opt. Express 15, 6768-6773 (2007).

144. L. A. Surin, B. S. Dumesh, F. Lewen, D. A. Roth, V. P. Kostromin, F. S. Rusin, G. Winnewisser, and I. Pak, "Millimeter-wave intracavity-jet OROTRON-spectrometer for investigation of van der Waals complexes,” Rev. Sci. Instrum. 72, 2535-2542 (2001).

145. G. Cazzoli, L. Cludi, C. Degli Esposti, and L. Dore, "Lamb-dip absorption spectroscopy in the far infrared region using a laser sideband spectrometer," J. Mol. Spectrosc. 151, 378-383 (1992).

146. M. Bellini, P. De Natale, and M. Inguscio, "Progress in the far-infrared precision spectroscopy," Laser Phys. 4, 408-411 (1994).

147. P. De Natale, L. Gianfrani, S. Viciani, and M. Inguscio Spectroscopic observation of the Faraday effect in the far infrared Optics Letters Vol. 22, pp. 1896-1898 (1997)

148. R. Braakman and G. A. Blake, "Principles and promise of FabryPerot resonators at terahertz frequencies," J.Appl. Phys. 109, 063102 (2011). 
149. A. Campa, L. Consolino, M. Ravaro, D. Mazzotti, M. S. Vitiello, S. Bartalini, and P. De Natale, "High-Q resonant cavities for terahertz quantum cascade lasers," Opt. Express 23, 3751-3761 (2015)

150. L. Carr, D. DeMille, R. Krems and J. Ye, Cold and ultracold molecules: science, technology and applications, New J. Phys. 11055049 (2009)

151. G. Quéméner and P.S. Julienne, Ultracold Molecules under Control!, Chem. Rev., 1124949 (2012)

152. M.H.G. de Miranda, A. Chotia, B. Neyenhuis, D. Wang, G. Quéméner, S. Ospelkaus, J.L. Bohn, J. Ye and D.S. Jin, Controlling the quantum stereodynamics of ultracold bimolecular reactions, Nature Phys., 7502 (2011)

153. S. Ospelkaus, K.-K. Ni, D. Wang, M.H.G. de Miranda, B. Neyenhuis, G. Quéméner, P.S. Julienne, J.L. Bohn, D.S. Jin and J. Ye, Quantum-State Controlled Chemical Reactions of Ultracold PotassiumRubidium Molecules, Science, 327853 (2010)

154. K.R.A. Hazzard, B. Gadway, M. Foss-Feig, B. Yan, S.A. Moses, J.P. Covey, N.Y. Yao, M.D. Lukin, J. Ye, D.S. Jin and A.M. Rey, Many-Body Dynamics of Dipolar Molecules in an Optical Lattice, Phys. Rev. Lett. 113195302 (2014)

155. J.D. Weinstein, R. de Carvalho, T. Guillet, B. Friedrich and J.M. Doyle, Magnetic trapping of calcium monohydride molecules at millikelvin temperatures, Nature 395148 (1998)

156. D. Patterson and J.M. Doyle, Cooling molecules in a cell for FTMW spectroscopy, Mol. Phys. 1101757 (2012)

157. S.E. Maxwell, N. Brahms, R. de Carvalho, D.R. Glenn, J.S. Helton, S.V. Nguyen, D. Patterson, J. Petricka, D. DeMille and J.M. Doyle, High-Flux Beam Source for Cold, Slow Atoms or Molecules, Phys. Rev. Lett. $95173201(2005)$

158. L.D. van Buuren, C. Sommer, M. Motsch, S. Pohle, M. Schenk, J. Bayerl, P.W.H. Pinkse and G. Rempe, Electrostatic Extraction of Cold Molecules from a Cryogenic Reservoir, Phys. Rev. Lett. 102033001 (2009)

159. D. Patterson, M. Schnell and J.M. Doyle, Enantiomer-specific detection of chiral molecules via microwave spectroscopy, Nature 497475 (2013)

160. B. Spaun, P.B. Changala, D. Patterson, B.J. Bjork, O.H. Heckl, J.M. Doyle and J. Ye, Continuous probing of cold complex molecules with infrared frequency comb spectroscopy, Nature 533517 (2016)

161. The ACME Collaboration, J. Baron, W.C. Campbell, D. DeMille, J. M. Doyle, G. Gabrielse, Y.V. Gurevich, P.W. Hess, N.R. Hutzler, E. Kirilov, I. Kozyryev, B.R. O’Leary, C.D. Panda, M.F. Parsons, E.S. Petrik, B. Spaun, A.C. Vutha and A.D. West, Order of Magnitude Smaller Limit on the Electric Dipole Moment of the Electron, Science 3436168 (2013)

162. L. Santamaria, V. Di Sarno, P. De Natale, M. De Rosa, M. Inguscio, S. Mosca, I. Ricciardi, D. Calonico, F. Levi and P. Maddaloni, Phys. Chem. Chem. Phys., 18, 16715 (2016)

163. G. Scoles, F. Torello, Supersonic molecular beams production and temperature distribution in free expanding jets, Meccanica, 3 20-27 (1968)

164. S.Y.T. van der Meerakker, H.L. Bethlem, N. Vanhaecke and G. Meijer, Manipulation and Control of Molecular Beams, Chem. Rev. 1124828 (2012)

165. M. Lemeshko, R.V. Krems, J.M. Doyle and S. Kais, Manipulation of molecules with electromagnetic fields, Mol. Phys. 1111648 (2013)

166. H.L. Bethlem, G. Berden and G. Meijer, Decelerating Neutral Dipolar Molecules, Phys. Rev. Lett. 831558 (1999)

167. H.L. Bethlem, G. Berden, F.M.H. Crompvoets, R.T. Jongma, A.J.A. van Roij and G. Meijer, Electrostatic trapping of ammonia molecules, Nature 406491 (2000)

168. N. Vanhaecke, U. Meier, M. Andrist, B.H. Meier and F. Merkt, Multistage Zeeman deceleration of hydrogen atoms, Phys. Rev. A 75 031402(R) (2007)

169. S.A. Meek, H.L. Bethlem, H. Conrad and G. Meijer, Trapping Molecules on a Chip in Traveling Potential Wells, Phys. Rev. Lett. 100153003 (2008)

170. A. Osterwalder, S.A. Meek, G. Hammer, H. Haak and G. Meijer, Deceleration of neutral molecules in macroscopic traveling traps, Phys. Rev. A 81 051401(R) (2010)

171. J. van Veldhoven, J. Küpper, H.L. Bethlem, B. Sartakov, A.J.A. van Roij and G. Meijer, Decelerated molecular beams for high-resolution spectroscopy, Eur. Phys. J. D 31337 (2004)

172. E.R. Hudson, H.J. Lewandowski, B.C. Sawyer and J. Ye, Cold Molecule Spectroscopy for Constraining the Evolution of the Fine Structure Constant, Phys. Rev. Lett. 96143004 (2006)

173. B.L. Lev, E.R. Meyer, E.R. Hudson, B.C. Sawyer, J.L. Bohn and J. Ye, OH hyperfine ground state: From precision measurement to molecular qubits, Phys. Rev. A 74 061402(R) (2006)

174. B.K. Stuhl, B.C. Sawyer, D. Wang and J. Ye, Magneto-optical Trap for Polar Molecules, Phys. Rev. Lett. $101243002(2008)$

175. E.S. Shuman, J.F. Barry, D.R. Glenn and D. DeMille, Radiative Force from Optical Cycling on a Diatomic Molecule, Phys. Rev. Lett. 103223001 (2009) 
176. E.S. Shuman, J.F. Barry and D. DeMille, Laser cooling of a diatomic molecule, Nature 467820 (2010)

177. J.F. Barry, E.S. Shuman, E.B. Norrgard and D. DeMille, Laser Radiation Pressure Slowing of a Molecular Beam, Phys. Rev. Lett. 108103002 (2012)

178. J.F. Barry, D.J. McCarron, E.B. Norrgard, M.H. Steinecker and D. DeMille, Magneto-optical trapping of a diatomic molecule, Nature 512286 (2014)

179. M.T. Hummon, M. Yeo, B.K. Stuhl, A.L. Collopy, Y. Xia and J. Ye, 2D Magneto-optical trapping of diatomic molecules, Phys. Rev. Lett. 110143001 (2013)

180. V. Zhelyazkova, A. Cournol, T.E. Wall, A. Matsushima, J.J. Hudson, E.A. Hinds, M.R. Tarbutt and B.E. Sauer, Laser cooling and slowing of CaF molecules, Phys. Rev. A 89053416 (2014)

181. M. Lombardi, The use of GPS disciplined oscillators as primary frequency standards for calibration and metrology laboratories, NCSLI Measure J. Meas. Sci. 3 (3), 56-65 (2008).

182. A. D. Ludlow, M. M. Boyd, J. Ye, and P. O. Schmidt, “Optical atomic clocks,” Rev. Mod. Phys. 87, 637701 (2015).

183. I. Ushijima, M. Takamoto, M. Das, T. Ohkubo, and H. Katori, "Cryogenic optical lattice clocks," Nature Photon. 9, 185-189 (2015).

184. B. J. Bloom, T. L. Nicholson, J. R. Williams, S. L. Campbell, M. Bishof, X. Zhang, W. Zhang, S. L. Bromley and J. Ye, "An optical lattice clock with accuracy and stability at the $10^{-18}$ level," Nature 506, 71-75 (2014).

185. N. Hinkley, J. A. Sherman, N. B. Phillips, M. Schioppo, N. D. Lemke, K. Beloy, M. Pizzocaro, C. W. Oates, and A. D. Ludlow, "An atomic clock with $10^{-18}$ instability," Science 341, 1215-1218 (2013).

186. D. Calonico, M. Inguscio, and F. Levi, "Light and the distribution of time," EPL 110, 40001 (2015).

187. S. Droste, F. Ozimek, T. Udem, K. Predehl, T. W. Hänsch, H. Schnatz, G. Grosche, and R. Holzwarth, "Optical-frequency transfer over a single-span 1840 km fiber link," Phys. Rev. Lett. 111, 110801 (2013).

188. D. Calonico, E. K. Bertacco, C. E. Calosso, C. Clivati, G. A. Costanzo, M. Frittelli, A. A. Godone Mura, N. Poli, D. V. Sutyrin, G. M. Tino, M. E. Zucco, and F. Levi, "High-accuracy coherent optical frequency transfer over a doubled 642-km fiber link," Appl. Phys. B 117, 979-986 (2014).

189. O. Lopez, A. Haboucha, B. Chanteau, C. Chardonnet, A. Amy-Klein, and G. Santarelli, "Ultra-stable long distance optical frequency distribution using the Internet fiber network," Opt. Express 20, 23518-23526 (2012).

190. M. Fujieda, M. Kumagai, S. Nagano, A. Yamaguchi, H. Hachisu, and T. Ido, "All-optical link for direct comparison of distant optical clocks," Opt. Express 19, 16498-16507 (2011).

191. A. Yamaguchi, M. Fujieda, M. Kumagai, H. Hachisu, S. Nagano, Y. Li, T. Ido, T. Takano, M. Takamoto, and H. Katori, "Direct comparison of distant optical lattice clocks at the $10^{-16}$ uncertainty," Appl. Phys. Express 4, 082203 (2011).

192. A. Matveev, C. G. Parthey, K. Predehl, J. Alnis, A. Beyer, R. Holzwarth, T. Udem, T. Wilken, N. Kolachevsky, M. Abgrall, D. Rovera, C. Salomon, P. Laurent, G. Grosche, O. Terra, T. Legero, H. Schnatz, S. Weyers, B. Altschul, and T. W. Hänsch, "Precision measurement of the hydrogen 1S2S frequency via a 920-km fiber link,” Phys. Rev. Lett. 110, 230801 (2013).

193. C. Clivati, G. A. Costanzo, M. Frittelli, F. Levi, A. Mura, M. Zucco, R. Ambrosini, C. Bortolotti, F. Perini, M. Roma, and D. Calonico, "A coherent fiber link for very long baseline interferometry," IEEE Trans. Ultrason. Ferroelectr. Freq. Control 62, 1907-1912 (2015).

194. S. Droste, C. Grebing, J. Leute, S. M. F. Raupach, A. Matveev, T. W. Hänsch, A. Bauch, R. Holzwarth, and G. Grosche, "Characterization of a 450-km baseline GPS carrier-phase link using an optical fiber link," New J. Phys. 17, 083044 (2015).

195. C. Lisdat, G. Grosche, N. Quintin, C. Shi, S.M.F. Raupach, C. Grebing, D. Nicolodi, F. Stefani, A. AlMasoudi, S. Dörscher, S. Häfner, J.-L. Robyr, N. Chiodo, S. Bilicki, E. Bookjans, A. Koczwara, S. Koke, A. Kuhl, F. Wiotte, F. Meynadier, E. Camisard, M. Abgrall, M. Lours, T. Legero, H. Schnatz, U. Sterr, H. Denker, C. Chardonnet, Y. Le Coq, G. Santarelli, A. Amy-Klein, R. Le Targat, J. Lodewyck, O. Lopez, and P.-E. Pottie, A clock network for geodesy and fundamental science, Nature Communications 712443 (2016).

196. P. Morzyński, M. Bober, D. Bartoszek-Bober, J. Nawrocki, P. Krehlik, Ł. Śliwczyński, M. Lipiński, P. Masłowski, A. Cygan, P. Dunst, M. Garus, D. Lisak, J. Zachorowski, W. Gawlik, C. Radzewicz, R. Ciuryło, M. Zawada, Absolute measurement of the ${ }^{1} \mathrm{~S}_{0}-{ }^{3} \mathrm{P}_{0}$ clock transition in neutral ${ }^{88} \mathrm{Sr}$ over the 330 km-long stabilized fibre optic link, Sci. Rep. 5, 17495 (2015).

197. A. Bjerhammar, "On a relativistic geodesy," B. Geod. 59, 207-220 (1985).

198. B. Argence, B. Chanteau, O. Lopez, D. Nicolodi, M. Abgrall, C. Chardonnet, C. Daussy, B. Darquié, Y. Le Coq, and A. Amy-Klein, "Quantum cascade laser frequency stabilization at the sub-Hz level," Nature Photon. 9, 456-460 (2015).

199. C. Clivati, G. Cappellini, L.F. Livi, F. Poggiali, M. Siciliani de Cumis, M. Mancini, G. Pagano, M. Frittelli, A. Mura, G.A. Costanzo, F. Levi, D. Calonico, L. Fallani, J. Catani, and M. Inguscio, Measuring absolute 
frequencies beyond the GPS limit via long-haul optical frequency dissemination, Optics Express, 24, 11865-11875 (2016)

200. M.A. Belkin, F. Capasso, F. Xie, A. Belyanin, M. Fischer, A. Wittmann, and J. Faist, Room temperature terahertz quantum cascade laser source based on intracavity difference-frequency generation, Applied Physics Letters, 92, 201101 (2008)

201. M. Razeghi, Q.Y. Lu, N. Bandyopadhyay, W. Zhou, D. Heydari, Y. Bai, S. Slivken, Quantum cascade lasers: from tool to product. Opt. Express 23, 8462 (2015).

202. M.A. Belkin, F. Capasso, New frontiers in quantum cascade lasers: high performance room temperature terahertz sources, Phys. Scr. 90, 118002 (2015).

203. Q.Y. Lu, N. Bandyopadhyay, S. Slivken, Y. Bai, M. Razeghi, Widely-tuned room temperature terahertz quantum cascade laser sources based on difference frequency generation, Appl. Phys. Lett. 101, 251121 (2012).

204. Q. Y. Lu, N. Bandyopadhyay, S. Slivken, Y. Bai, and M. Razeghi High performance terahertz quantum cascade laser sources based on intracavity difference frequency generation Optics Express Vol. 21, pp. 968-973 (2013)

205. K. Vijayraghavan, Y. Jiang, M. Jang, A. Jiang, K. Choutagunta, A. Vizbaras, F. Demmerle, G. Boehm, M. C. Amann, M.A. Belkin, Broadly tunable terahertz generation in mid-infrared quantum cascade lasers. Nat. Commun. 4, 2021 (2013).

206. S. Jung, A. Jiang, Y. Jiang, K. Vijayraghavan, X. Wang, M. Troccoli, M. A. Belkin, Broadly tunable monolithic room-temperature terahertz quantum cascade laser sources. Nature Commun. 5, 4267 (2014).

207. Q.Y. Lu, S. Slivken, N. Bandyopadhyay, Y. Bai, M. Razeghi, Widely tunable room temperature semiconductor terahertz source. Appl. Phys. Lett. 105, 201102 (2014).

208. Q.Y. Lu, N. Bandyopadhyay, S. Slivken, Y. Bai, M. Razeghi, Continuous operation of a monolithic semiconductor terahertz source at room temperature. Appl. Phys. Lett. 104, 221105 (2014).

209. Q. Lu, D. Wu, S. Sengupta, S. Slivken, M. Razeghi, Sci. Rep. 643806 (2017)

210. D. Burghoff, T.-Y. Kao, N. Han, C. Wang Chan, X. Cai, Y. Yang, D. Hayton, J.-R. Gao, J.L. Reno, Q. Hu, Terahertz laser frequency combs, Nature Photonics 8, 462 (2014).

211. M. Rösch, G. Scalari, M. Beck and J. Faist, Octave-spanning semiconductor laser, Nature Photonics, 942 (2015)

212. J.B. Khurgin, Y. Dikmelik, A. Hugi, J. Faist, Coherent frequency combs produced by self frequency modulation in quantum cascade lasers, Appl. Phys. Lett. 104, 081118 (2014).

213. S. Barbieri, M. Ravaro, P. Gellie, G. Santarelli, C. Manquest, C. Sirtori, S.P. Khanna, E.H. Linfield, A. Giles Davies, Coherent sampling of active mode-locked terahertz quantum cascade lasers and frequency synthesis. Nature Photon. 5, 306-313 (2011).

214. M. Amanti, G. Scalari, R. Terazzi, M. Fischer, M. Beck, J. Faist, A. Rudra, P. Gallo and E. Kapon, Boundto-continuum terahertz quantum cascade laser with a single quantum well phonon extraction/injection stage, New J. Phys. 11, 125022 (2009).

215. G. Scalari, C. Walther, M. Fischer, R. Terazzi, H. Beere, D. Ritchie, J. Faist, THz and sub-THz quantum cascade lasers. Laser Photon. Rev. 3, 45-46 (2009).

216. J. Faist, F. Capasso,D.L. Sivco, C. Sirtori, A.L. Hutchinson, A.Y. Cho, Quantum cascade laser. Science 264, 553-556 (1994).

217. C. Gmachl, D.L. Sivco, R. Colombelli, F. Capasso, A.Y. Cho, Ultra-broadband semiconductor laser. Nature 415, 883-887 (2002).

218. J.R. Freeman, O.P. Marshall, H.E. Beere, D.A. Ritchie, Electrically switchable emission in terahertz quantum cascade lasers. Opt. Express 16, 19830 (2008).

219. J.R. Freeman, J. Madéo, A. Brewer, S. Dhillon, O.P. Marshall, N. Jukam, D. Oustinov, J. Tignon, H.E. Beere, and D.A. Ritchie, Dual wavelength emission from a terahertz quantum cascade laser. Appl. Phys. Lett. 96, 051120 (2010).

220. S.P. Khanna, M. Salih, P. Dean, A.G. Davies, E.H. Linfield, Electrically tunable terahertz quantumcascade laser with a heterogeneous active region. Appl. Phys. Lett. 95, 181101 (2009). 